\title{
Spectral correlation analysis and antimicrobial activities of some 2,4-dimethoxy phenyl chalcones
}

\author{
S. John Joseph ${ }^{1}$, R. Arulkumaran ${ }^{1}$, D. Kamalakkannan ${ }^{1}$, S. P. Sakthinathan ${ }^{1}$, \\ R. Sundararajan ${ }^{1}$, R. Suresh ${ }^{1}$, S. Vijayakumar ${ }^{1}$, K. Ranganathan ${ }^{1}$, \\ N. Kalyanasundaram, G. Vanangamudi ${ }^{1, *}$, G. Thirunarayanan ${ }^{2}$ \\ ${ }^{1}$ PG \& Research Department of Chemistry, Government Arts College, C-Mutlur, \\ Chidambaram - 608102, India. \\ ${ }^{2}$ Department of Chemistry, Annamalai University, Annamalainagar - 608002, India. \\ *E-mail address: drgvsibi@gmail.com
}

\begin{abstract}
A series of 2,4-dimethoxy phenyl chalcones have been synthesized by Crossed-Aldol condensation of 2,4-dimethoxy phenyl and various substituted benzaldehydes. The purities of these chalcones have been checked by their physical constants, UV, IR, NMR and MASS spectral data. The spectral data of these chalcones have been correlated with Hammett sigma constants, F and R parameters using single and multi-linear regression analysis. From the results of statistical analysis, the effects of substituents on the spectral group frequencies have been discussed. The anti-microbial activities of these chalcones have been evaluated using Bauer-Kirby method.
\end{abstract}

Keywords: substituted styryl 2,4-dimethoxy phenyl ketones; UV spectra; IR spectra; NMR spectra; substituent effects; antimicrobial activities

\section{INTRODUCTION}

The $\sigma, \beta$ unsaturated ketones are called as enones. They possess carbonyl moiety and it was bonded with aryl or alkyl groups in both sides. They are very important molecules for synthesising heterocycles and possess multipronged activities [1]. Various solvent assisted and green synthetic methodologies have been reported for the synthesis of aryl enones. The Crossed-Aldol [2], Claisen Schmidt [3], Knovenagal [4] and Wittig [5] reactions are important for the synthesis of enones. Many catalyst were used for proceedings the above said reactions namely, EtOH-NaOH [6], MeOH-KOH [7], EtOH-KOH [8], $\mathrm{MgCl}_{2}$ [5], silicasulphuric acid [9], anhydrous zinc chloride [10], clay [11], Hydrotalcite [12], ground chemistry catalysts-grinding the reactants with sodium hydroxide [13], aqueous alkali in lower temperature [14], solid sulphonic acid from bamboo [15], barium hydroxide [16] anhydrous sodium bicarbonate [17], microwave assisted synthesis [18], fly-ash:water [19], fly-ash: $\mathrm{H}_{2} \mathrm{SO}_{4}$ [20], fly-ash:PTS [21], $\mathrm{NaOH}-\mathrm{CTABr}$ [22], $\mathrm{SiO}_{2}-\mathrm{H}_{3} \mathrm{PO}_{4}$ [23], $\mathrm{SOCl}_{2}$ [24] and sulfated titania [25]. The spectral data of these E- chalcones were useful for prediction of ground state equilibration like s-cis and s-trans conformers. Recently, Janaki et al., [23] have elaborately studied the effects of substituent on the ultraviolet absorption $(\lambda \max , \mathrm{nm})$, infrared group frequencies $\left(v, \mathrm{~cm}^{-1}\right)$ and NMR chemical shifts $(\delta, \mathrm{ppm})$ of $\sigma, \beta$ - proton and 
carbons of 4-ethoxy phenyl chalcones and benzimidazole chalcones. In their studies, they observed satisfactory and good correlations for each spectral data with Hammett substituent constants, $\mathrm{F}$ and $\mathrm{R}$ parameters. Chalcones possess various multipronged activities such as antimicrobial [26], antidepressants [27], antiplosmodial [28], anti-aids [29] and insect antifeedant activities $[13,22]$. In the present investigation, the authors have synthesized a series of some substituted styryl 2,4-dimethoxy phenyl ketones, studied the spectral correlations and to evaluate the antimicrobial activities using Bauer-Kirby [30] method.

\section{EXPERIMENTAL}

\section{1. General}

All chemicals used were purchased from Sigma-Aldrich and E-Merck chemical companies. Melting points of all chalcones were determined in open glass capillaries on Mettler FP51 melting point apparatus and are uncorrected. The ultraviolet spectrophotometer was utilized for recording the absorption maxima $(\lambda \max , \mathrm{nm})$, of all chalcones in spectral grade solvent. Infrared spectra $\left(\mathrm{KBr}, 4000-400 \mathrm{~cm}^{-1}\right)$ were recorded AVATAR-300 Fourier transform spectrophotometer. BRUKER AVANCE III-500MHz NMR spectrometer was used for recording NMR spectra operating at $500 \mathrm{MHz}$ for ${ }^{1} \mathrm{H}$ spectra and $125.46 \mathrm{MHz}$ for ${ }^{13} \mathrm{C}$ spectra in $\mathrm{CDCl}_{3}$ solvent using TMS as internal standard. Mass spectra of all chalcones were recorded on SHIMADZU spectrometer using chemical ionization technique.

\section{2. Synthesis of Chalcones}

All substituted 2,4-dimethoxy phenyl chalcones were synthesized by procedure published in literature [19,31]. An equi-molar quantities of 2,4-dimethoxy acetophenone (2 mmol) substituted benzaldehydes $(2 \mathrm{mmol})$, Fly-ash $(0.5 \mathrm{~g})$ and $20 \mathrm{~mL}$ of water (Scheme 1) were refluxed for $4 \mathrm{~h}$. The completion of the reaction was monitored by thin layer chromatography. After completion of the reaction, the solid product was separated by filtration. Further the product was purified by column chromatography using dichloromethane and ethyl acetate mixture as eluent. The physical constants, analytical and mass fragments were presented in Table 1 .

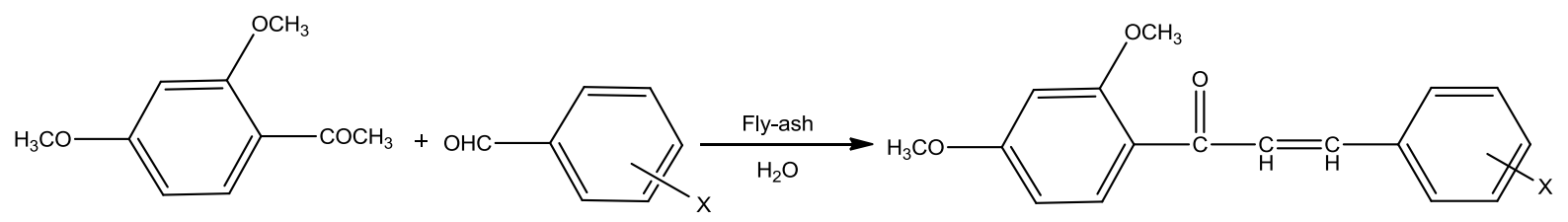

where $\mathrm{X}=\mathrm{H}, 4-\mathrm{Br}, 2-\mathrm{Cl}, 3-\mathrm{Cl}, 4-\mathrm{Cl}, 4-\mathrm{F}, 2-\mathrm{OCH}_{3}, 3-\mathrm{NO}_{2}, 4-\mathrm{NO}_{2}$

Scheme 1. Synthesis of substituted styryl 2, 4-dimethoxy phenyl ketones. 
Table 1. Physical constants and mass spectral data of substituted styryl 2,4-dimethoxy phenyl ketones.

\begin{tabular}{|c|c|c|c|c|c|c|}
\hline Entry & $\mathrm{X}$ & $\mathrm{M} . \mathrm{F}$. & $\mathrm{M} . \mathrm{W}$. & $\begin{array}{c}\text { Yield } \\
(\%)\end{array}$ & m.p. $\left({ }^{\circ} \mathrm{C}\right)$ & Mass (m/z) \\
\hline 1 & $\mathrm{H}$ & $\mathrm{C}_{17} \mathrm{H}_{16} \mathrm{O}_{3}$ & 268 & 95 & $\begin{array}{c}78-80 \\
(80.5-81)[32]\end{array}$ & $268[\mathrm{M}+]$ \\
\hline 2 & $4-\mathrm{Br}$ & $\mathrm{C}_{17} \mathrm{H}_{15} \mathrm{BrO}_{3}$ & 347 & 90 & $133-135$ & $347[\mathrm{M}+], 349[\mathrm{M} 2+]$ \\
\hline 3 & $2-\mathrm{Cl}$ & $\mathrm{C}_{17} \mathrm{H}_{15} \mathrm{ClO}_{3}$ & 302 & 89 & $\begin{array}{c}118-120 \\
(119-120)[33]\end{array}$ & $302[\mathrm{M}+], 304[\mathrm{M} 2+]$ \\
\hline 4 & $3-\mathrm{Cl}$ & $\mathrm{C}_{17} \mathrm{H}_{15} \mathrm{ClO}_{3}$ & 302 & 88 & $115-116$ & $302[\mathrm{M}+], 304[\mathrm{M} 2+]$ \\
\hline 5 & $4-\mathrm{Cl}$ & $\mathrm{C}_{17} \mathrm{H}_{15} \mathrm{ClO}_{3}$ & 302 & 88 & $\begin{array}{c}117-119 \\
(118-120)[33]\end{array}$ & $302[\mathrm{M}+], 304[\mathrm{M} 2+]$ \\
\hline 6 & $4-\mathrm{F}$ & $\mathrm{C}_{17} \mathrm{H}_{15} \mathrm{FO}_{3}$ & 286 & 87 & $\begin{array}{c}96-98 \\
(98)[34]\end{array}$ & $286[\mathrm{M}+], 288[\mathrm{M} 2+]$ \\
\hline 7 & $2-\mathrm{OCH}_{3}$ & $\mathrm{C}_{18} \mathrm{H}_{18} \mathrm{O}_{4}$ & 298 & 92 & $\begin{array}{c}105-106 \\
(106-107)[35]\end{array}$ & $298[\mathrm{M}+]$ \\
\hline 8 & $3-\mathrm{NO}_{2}$ & $\mathrm{C}_{17} \mathrm{H}_{15} \mathrm{NO}_{5}$ & 313 & 86 & $\begin{array}{c}185-187 \\
190-191 \\
(191)[36]\end{array}$ & $313[\mathrm{M}+]$ \\
\hline 9 & $4-\mathrm{NO}_{2}$ & $\mathrm{C}_{17} \mathrm{H}_{15} \mathrm{NO}_{5}$ & 313 & 85 & $313+\mathrm{M}+]$ \\
\hline
\end{tabular}

\section{RESULTS AND DISCUSSION}

In the present study the authors have studied the effects of substituents on the spectral data such as ultraviolet absorption maxima $(\lambda \max , \mathrm{nm})$, infrared spectral carbonyl and deformation modes $\left(v, \mathrm{~cm}^{-1}\right)$, the chemical shifts $(\delta, \mathrm{ppm})$ of $\alpha, \beta$ protons, and carbonyl carbons of synthesized 2,4-dimethoxy phenyl chalcones with Hammett substituent constants, $\mathrm{F}$ and $\mathrm{R}$ parameters using single and multi-linear regression analysis for predicting the reactivity on the group frequencies.

\section{1. UV spectral study}

The measured ultraviolet absorption maxima $(\lambda \max , \mathrm{nm})$ of all 2,4-dimethoxy phenyl chalcones were tabulated in Table 2 . These values are correlated [1,2,6,10,19,21,23-25,28,37] with Hammett substituent constants, $\mathrm{F}$ and $\mathrm{R}$ parameters using single and multi-linear regression analysis. While seeking Hammett correlation, involving UV absorption maximum values, the form of the Hammett equation employed is as given in equation.

$$
\lambda \max =\rho \sigma+\lambda_{0}
$$

The results of statistical analysis are presented in Table 3. From the Table 3, the correlation of absorption maxima $(\lambda \max , \mathrm{nm})$ with $\sigma$ and $\sigma^{+}$parameter is satisfactory. The remaining Hammett substituent constants, $\mathrm{F}$ and $\mathrm{R}$ parameter were failing in correlation. This 
is due to the inductive and resonance effects of substituents incapable for predicting the reactivity on the absorption and is associated with the resonance conjugated structure as shown in Fig. 1. In single parameter correlation, the Hammett substituents constants not obeyed in the regression. While seeking these parameters in multi-regression, with $\mathrm{F}$ and $\mathrm{R}$ Swain-Lupton's [37] constants, they gave satisfactory correlations. The multi correlation equations are given in (2) and (3).

$$
\begin{gathered}
\lambda \max (\mathrm{nm})=310.89( \pm 12.776)-16.935( \pm 24.427) \sigma_{\mathrm{I}}-34.183( \pm 19.1018) \sigma_{\mathrm{R}} \\
(R=0.965, \mathrm{n}=9, \mathrm{P}>95 \%)
\end{gathered}
$$

$\lambda \max (\mathrm{nm})=312.72( \pm 11.184)-21.889( \pm 21.648) \mathrm{F}-32.685( \pm 18.371) \mathrm{R}$

$$
(R=0.963, \mathrm{n}=9, \mathrm{P}>95 \%)
$$

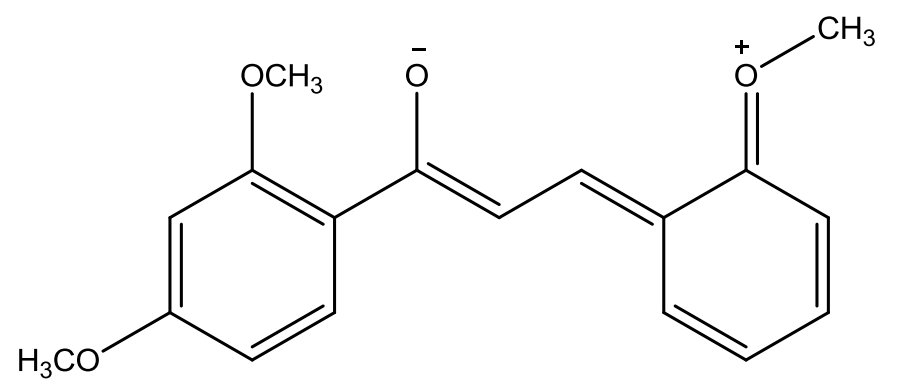

Fig. 1. The resonance- conjugated structure.

\begin{tabular}{|c|c|c|c|c|c|c|c|c|c|c|c|c|c|}
\hline$\underset{\Xi}{\stackrel{\Xi}{E}}$ & $\mathbf{X}$ & 气 & 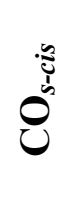 & $\overbrace{}^{\tilde{\Sigma}}$ & تิ & $\underbrace{8}$ & 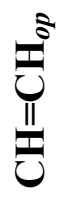 & $U_{0}^{\tilde{s}}$ & 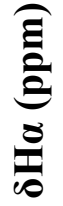 & 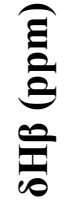 & 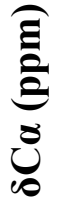 & 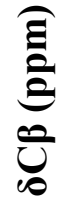 & 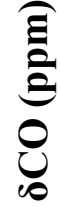 \\
\hline 1 & $I$ & $\begin{array}{l}\infty \\
\stackrel{\infty}{0} \\
\text { ô }\end{array}$ & $\begin{array}{l}\hat{\sigma} \\
\infty \\
\infty \\
0 \\
0\end{array}$ & $\begin{array}{l}\stackrel{a}{+} \\
\dot{n} \\
\infty \\
n \\
n\end{array}$ & $\begin{array}{l}\vec{\sim} \\
\stackrel{\sim}{\Xi} \\
ٍ\end{array}$ & 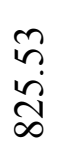 & 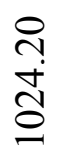 & $\begin{array}{l}\stackrel{f}{\sim} \\
\hat{n} \\
n\end{array}$ & $\stackrel{m}{\mathfrak{n}}$ & 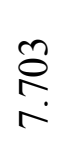 & $\begin{array}{l}\text { ָ̃ } \\
\text { ㄱ }\end{array}$ & $\begin{array}{l}\tilde{n} \\
\stackrel{n}{n} \\
n\end{array}$ & 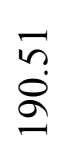 \\
\hline 2 & $\dot{\bar{p}}$ & 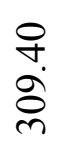 & $\begin{array}{l}ت \\
0 \\
0 \\
0\end{array}$ & $\begin{array}{l}n \\
\infty \\
0 \\
\check{n} \\
\end{array}$ & $\begin{array}{l}0 \\
⿱ n \\
\infty \\
\stackrel{N}{J} \\
=\end{array}$ & 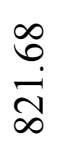 & 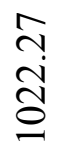 & $\begin{array}{c}\tilde{a} \\
\tilde{y} \\
\tilde{n}\end{array}$ & $\stackrel{m}{n}$ & $\begin{array}{l}\hat{\sigma} \\
\stackrel{\sim}{*}\end{array}$ & $\begin{array}{l}\text { ठ } \\
\text { ̇. }\end{array}$ & $\begin{array}{l}\stackrel{q}{+} \\
\dot{\sim} \\
\stackrel{+}{-}\end{array}$ & $\begin{array}{l}\text { ᄋे } \\
\dot{8}\end{array}$ \\
\hline 3 & $\begin{array}{l}u \\
\sim \\
\sim\end{array}$ & $\frac{\stackrel{+}{+}}{\stackrel{i}{\circ}}$ & $\begin{array}{l}2 \\
\text { } \\
8 \\
8\end{array}$ & $\begin{array}{l}\hat{\sigma} \\
\vec{n} \\
\tilde{n}\end{array}$ & $\begin{array}{l}8 \\
\Xi \\
\equiv \\
\equiv\end{array}$ & $\begin{array}{l}\stackrel{8}{0} \\
\stackrel{\text { }}{\infty}\end{array}$ & $\begin{array}{l}\stackrel{0}{q} \\
\stackrel{0}{0} \\
0\end{array}$ & $\begin{array}{l}\vec{\sim} \\
\hat{n} \\
\stackrel{n}{n}\end{array}$ & $\frac{\sim}{n}$ & $\begin{array}{l}\hat{b} \\
\varnothing \\
\infty\end{array}$ & ๙ே & $\begin{array}{l}\hat{\infty} \\
\dot{m} \\
\ddot{n}\end{array}$ & $\begin{array}{l}\text { กิ } \\
\stackrel{8}{\Omega}\end{array}$ \\
\hline
\end{tabular}

Table 2. The ultraviolet absorption maxima $(\lambda \max , \mathrm{nm})$, infrared absorptions $\left(v, \mathrm{~cm}^{-1}\right)$ and NMR chemical shifts $(\delta, \mathrm{ppm})$ of substituted styryl 2,4-dimethoxy phenyl ketones. 


\begin{tabular}{|c|c|c|c|c|c|c|c|c|c|c|c|c|c|}
\hline 4 & 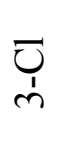 & 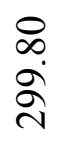 & 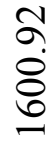 & 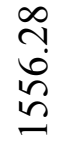 & $\begin{array}{l}5 \\
8 \\
8 \\
=\end{array}$ & $\frac{n}{\infty}$ & 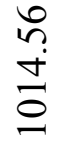 & $\begin{array}{l}\stackrel{\infty}{\sim} \\
\stackrel{+}{\sim} \\
\stackrel{n}{n}\end{array}$ & $\begin{array}{l}0 \\
\text { m} \\
r\end{array}$ & $\underset{r}{\frac{\infty}{\sigma}}$ & $\frac{\Xi}{2}$ & 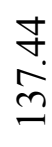 & $\begin{array}{l}\curvearrowleft \\
\varrho \\
\infty\end{array}$ \\
\hline 5 & $\overrightarrow{\dot{J}^{\prime}}$ & $\frac{8}{\bar{m}}$ & $\begin{array}{l}\infty \\
\infty \\
\infty \\
0 \\
\sigma\end{array}$ & $\begin{array}{l}n \\
\infty \\
\hat{n} \\
n \\
n\end{array}$ & 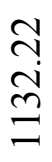 & $\frac{n}{n}$ & 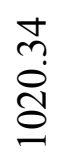 & $\begin{array}{l}8 \\
\text { i } \\
\text { in }\end{array}$ & $\frac{a}{n}$ & \begin{tabular}{l}
$n$ \\
\multirow{v}{*}{} \\
\end{tabular} & $\begin{array}{l}\text { ¿ } \\
\text { ㄱ. }\end{array}$ & $\begin{array}{l}\stackrel{m}{n} \\
\stackrel{n}{n}\end{array}$ & $\underset{\check{g}}{\beth}$ \\
\hline 6 & $\frac{I}{\dot{H}}$ & $\begin{array}{l}8 \\
\infty \\
\infty \\
\infty \\
\text { D }\end{array}$ & $\begin{array}{l}0 \\
0 \\
0 \\
0\end{array}$ & 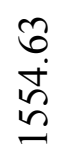 & 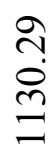 & 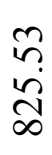 & 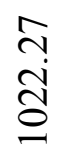 & \begin{tabular}{l}
$\tilde{n}$ \\
$\infty$ \\
$\infty$ \\
\multirow{\gamma}{*}{}
\end{tabular} & 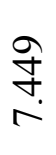 & $\underset{r}{\vec{\sigma}}$ & $\begin{array}{l} \pm \\
\stackrel{J}{J}\end{array}$ & $\stackrel{r}{\stackrel{n}{n}}$ & $\begin{array}{l}2 \\
\mathfrak{2} \\
\stackrel{8}{2}\end{array}$ \\
\hline 7 & $\begin{array}{l}\mathbf{I} \\
\stackrel{1}{0} \\
\stackrel{1}{N}\end{array}$ & $\begin{array}{l}\dot{8} \\
\dot{m} \\
\dot{m}\end{array}$ & $\stackrel{尺}{\stackrel{8}{8}}$ & $\begin{array}{l}\vec{\gamma} \\
\dot{0} \\
\stackrel{0}{n}\end{array}$ & $\begin{array}{l}8 \\
0 \\
0 \\
= \\
=\end{array}$ & \begin{tabular}{l}
\multirow{2}{n}{} \\
$\ddot{n}$ \\
$\infty$
\end{tabular} & $\begin{array}{l}\stackrel{̊}{+} \\
\stackrel{+}{ \pm}\end{array}$ & $\begin{array}{l}\hat{n} \\
\text { n} \\
\text { nn }\end{array}$ & $\stackrel{\circ}{\frac{O}{\sigma}}$ & $\underset{ن}{0}$ & $\begin{array}{l}n \\
0 \\
\\
\end{array}$ & \begin{tabular}{l}
$\infty$ \\
\multirow{2}{*}{} \\
$\stackrel{+}{ \pm}$
\end{tabular} & $\begin{array}{l}\infty \\
0 \\
\dot{8} \\
\Omega\end{array}$ \\
\hline 8 & $\begin{array}{l}\delta_{Z} \\
Z_{1}\end{array}$ & 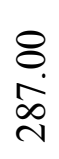 & $\begin{array}{l}n \\
\infty \\
i \\
\delta \\
0\end{array}$ & 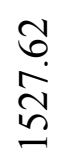 & 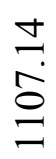 & $\begin{array}{l}\infty \\
\dot{0} \\
\infty\end{array}$ & $\begin{array}{l}\hat{n} \\
\dot{0} \\
0\end{array}$ & $\begin{array}{l}\vec{\sigma} \\
\stackrel{\sim}{*} \\
\dot{n}\end{array}$ & $\begin{array}{l}\text { నू } \\
n \\
r\end{array}$ & $\stackrel{\hat{2}}{\stackrel{2}{r}}$ & 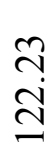 & $\begin{array}{l}0 \\
+ \\
\infty \\
\end{array}$ & $\begin{array}{l}\tilde{n} \\
\grave{\infty} \\
\infty\end{array}$ \\
\hline 9 & 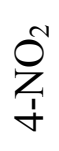 & 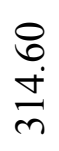 & 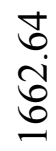 & $\begin{array}{l}n \\
0 \\
0 \\
n\end{array}$ & $\begin{array}{l}\tilde{a} \\
\text { ñ }\end{array}$ & $\frac{\tilde{n}}{n}$ & 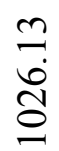 & $\begin{array}{l} \pm \\
\infty \\
n \\
n\end{array}$ & $\frac{N}{r}$ & $\underset{\infty}{ \pm}$ & $\frac{+}{n}$ & $\frac{n}{a}$ & $\begin{array}{l}\infty \\
\stackrel{1}{2} \\
\infty\end{array}$ \\
\hline
\end{tabular}

Table 3. Results of statistical analysis of ultraviolet absorption maxima $(\lambda \max , \mathrm{nm})$, infrared absorptions $\left(v, \mathrm{~cm}^{-1}\right)$ and NMR chemical shifts $(\delta, \mathrm{ppm})$ of substituted styryl 2,4-dimethoxy phenyl ketones with Hammett $\sigma, \sigma^{+}, \sigma_{\mathrm{I}}, \sigma_{\mathrm{R}}$ and $\mathrm{F}$ and R parameters.

\begin{tabular}{|c|c|c|c|c|c|c|c|}
\hline Frequency & Constants & $\mathbf{r}$ & $\mathbf{I}$ & $\rho$ & $\mathbf{s}$ & $\mathbf{n}$ & Correlated derivatives \\
\hline \multirow{5}{*}{$\lambda_{\max }$} & $\sigma$ & 0.906 & 316.19 & -28.59 & 11.97 & 8 & $\begin{array}{c}\mathrm{H}, 4-\mathrm{Br}, 2-\mathrm{Cl}, 3-\mathrm{Cl}, 4-\mathrm{Cl} \\
4-\mathrm{F}, 3-\mathrm{NO}_{2}, 4-\mathrm{NO}_{2}\end{array}$ \\
\hline & $\sigma^{+}$ & 0.907 & 316.11 & -26.66 & 11.49 & 8 & $\begin{array}{c}\mathrm{H}, 4-\mathrm{Br}, 2-\mathrm{Cl}, 3-\mathrm{Cl}, 4-\mathrm{Cl}, \\
4-\mathrm{F}, 2-\mathrm{OCH}_{3}, 3-\mathrm{NO}_{2}\end{array}$ \\
\hline & $\sigma_{\mathrm{I}}$ & 0.802 & 320.82 & -26.21 & 15.33 & 9 & $\begin{array}{c}\mathrm{H}, 4-\mathrm{Br}, 2-\mathrm{Cl}, 3-\mathrm{Cl}, 4-\mathrm{Cl}, 4-\mathrm{F}, \\
2-\mathrm{OCH}_{3}, 3-\mathrm{NO}_{2}, 4-\mathrm{NO}_{2}\end{array}$ \\
\hline & $\sigma_{\mathrm{R}}$ & 0.861 & 302.88 & -36.99 & 12.86 & 9 & $\begin{array}{c}\mathrm{H}, 4-\mathrm{Br}, 2-\mathrm{Cl}, 3-\mathrm{Cl}, 4-\mathrm{Cl}, 4-\mathrm{F}, \\
2-\mathrm{OCH}_{3}, 3-\mathrm{NO}_{2}, 4-\mathrm{NO}_{2}\end{array}$ \\
\hline & $\mathrm{F}$ & 0.820 & 318.40 & -20.56 & 15.55 & 9 & $\begin{array}{c}\mathrm{H}, 4-\mathrm{Br}, 2-\mathrm{Cl}, 3-\mathrm{Cl}, 4-\mathrm{Cl}, 4-\mathrm{F}, \\
2-\mathrm{OCH}_{3}, 3-\mathrm{NO}_{2}, 4-\mathrm{NO}_{2}\end{array}$ \\
\hline
\end{tabular}




\begin{tabular}{|c|c|c|c|c|c|c|c|}
\hline & $\mathrm{R}$ & 0.855 & 303.01 & -32.04 & 13.61 & 9 & $\begin{array}{c}\mathrm{H}, 4-\mathrm{Br}, 2-\mathrm{Cl}, 3-\mathrm{Cl}, 4-\mathrm{Cl}, 4-\mathrm{F}, \\
2-\mathrm{OCH}_{3}, 3-\mathrm{NO}_{2}, 4-\mathrm{NO}_{2}\end{array}$ \\
\hline \multirow{6}{*}{$\mathrm{CO}_{s-c i s}$} & $\sigma$ & 0.703 & 1616.74 & 25.48 & 28.72 & 9 & $\begin{array}{c}\mathrm{H}, 4-\mathrm{Br}, 2-\mathrm{Cl}, 3-\mathrm{Cl}, 4-\mathrm{Cl}, 4-\mathrm{F} \\
\text { 2- } \mathrm{OCH}_{3}, 3-\mathrm{NO}_{2}, 4-\mathrm{NO}_{2}\end{array}$ \\
\hline & $\sigma^{+}$ & 0.792 & 1617.69 & 20.40 & 29.05 & 9 & $\begin{array}{c}\mathrm{H}, 4-\mathrm{Br}, 2-\mathrm{Cl}, 3-\mathrm{Cl}, 4-\mathrm{Cl}, 4-\mathrm{F}, \\
\text { 2- } \mathrm{OCH}_{3}, 3-\mathrm{NO}_{2}, 4-\mathrm{NO}_{2}\end{array}$ \\
\hline & $\sigma_{\mathrm{I}}$ & 0.823 & 1605.88 & 38.50 & 29.25 & 9 & $\begin{array}{c}\mathrm{H}, 4-\mathrm{Br}, 2-\mathrm{Cl}, 3-\mathrm{Cl}, 4-\mathrm{Cl}, 4-\mathrm{F}, \\
2-\mathrm{OCH}_{3}, 3-\mathrm{NO}_{2}, 4-\mathrm{NO}_{2}\end{array}$ \\
\hline & $\sigma_{\mathrm{R}}$ & 0.908 & 1629.25 & 36.79 & 28.68 & 9 & $\begin{array}{c}\mathrm{H}, 4-\mathrm{Br}, 2-\mathrm{Cl}, 3-\mathrm{Cl}, 4-\mathrm{Cl}, 4-\mathrm{F}, \\
2-\mathrm{OCH}_{3}, 3-\mathrm{NO}_{2}, 4-\mathrm{NO}_{2}\end{array}$ \\
\hline & $\mathrm{F}$ & 0.714 & 1612.99 & 22.29 & 29.90 & 9 & $\begin{array}{c}\mathrm{H}, 4-\mathrm{Br}, 2-\mathrm{Cl}, 3-\mathrm{Cl}, 4-\mathrm{Cl}, 4-\mathrm{F} \\
\text { 2- } \mathrm{OCH}_{3}, 3-\mathrm{NO}_{2}, 4-\mathrm{NO}_{2}\end{array}$ \\
\hline & $\mathrm{R}$ & 0.906 & 1628.84 & 30.42 & 29.15 & 9 & $\begin{array}{c}\mathrm{H}, 4-\mathrm{Br}, 2-\mathrm{Cl}, 3-\mathrm{Cl}, 4-\mathrm{Cl}, 4-\mathrm{F}, \\
2-\mathrm{OCH}_{3}, 3-\mathrm{NO}_{2}, 4-\mathrm{NO}_{2}\end{array}$ \\
\hline \multirow{6}{*}{$\mathrm{CO}_{\text {s-trans }}$} & $\sigma$ & 0.819 & 1552.33 & -10.03 & 38.46 & 9 & $\begin{array}{c}\mathrm{H}, 4-\mathrm{Br}, 2-\mathrm{Cl}, 3-\mathrm{Cl}, 4-\mathrm{Cl}, 4-\mathrm{F}, \\
\text { 2- } \mathrm{OCH}_{3}, 3-\mathrm{NO}_{2}, 4-\mathrm{NO}_{2}\end{array}$ \\
\hline & $\sigma^{+}$ & 0.711 & 1552.52 & -10.23 & 38.40 & 9 & $\begin{array}{c}\mathrm{H}, 4-\mathrm{Br}, 2-\mathrm{Cl}, 3-\mathrm{Cl}, 4-\mathrm{Cl}, 4-\mathrm{F}, \\
2-\mathrm{OCH}_{3}, 3-\mathrm{NO}_{2}, 4-\mathrm{NO}_{2}\end{array}$ \\
\hline & $\sigma_{\mathrm{I}}$ & 0.905 & 1577.65 & -62.50 & 36.32 & 9 & $\begin{array}{c}\mathrm{H}, 4-\mathrm{Br}, 2-\mathrm{Cl}, 3-\mathrm{Cl}, 4-\mathrm{Cl}, 4-\mathrm{F}, \\
2-\mathrm{OCH}_{3}, 3-\mathrm{NO}_{2}, 4-\mathrm{NO}_{2}\end{array}$ \\
\hline & $\sigma_{\mathrm{R}}$ & 0.711 & 1552.64 & 16.33 & 38.40 & 9 & $\begin{array}{c}\mathrm{H}, 4-\mathrm{Br}, 2-\mathrm{Cl}, 3-\mathrm{Cl}, 4-\mathrm{Cl}, 4-\mathrm{F} \\
\text { 2- } \mathrm{OCH}_{3}, 3-\mathrm{NO}_{2}, 4-\mathrm{NO}_{2}\end{array}$ \\
\hline & $\mathrm{F}$ & 0.903 & 1571.77 & -48.80 & 36.88 & 9 & $\begin{array}{c}\mathrm{H}, 4-\mathrm{Br}, 2-\mathrm{Cl}, 3-\mathrm{Cl}, 4-\mathrm{Cl}, 4-\mathrm{F}, \\
\text { 2- } \mathrm{OCH}_{3}, 3-\mathrm{NO}_{2}, 4-\mathrm{NO}_{2}\end{array}$ \\
\hline & $\mathrm{R}$ & 0.745 & 1550.94 & 5.606 & 38.63 & 9 & $\begin{array}{c}\mathrm{H}, 4-\mathrm{Br}, 2-\mathrm{Cl}, 3-\mathrm{Cl}, 4-\mathrm{Cl}, 4-\mathrm{F}, \\
2-\mathrm{OCH}_{3}, 3-\mathrm{NO}_{2}, 4-\mathrm{NO}_{2}\end{array}$ \\
\hline \multirow{6}{*}{$\mathrm{CH}_{i p}$} & $\sigma$ & 0.905 & 1135.45 & -28.88 & 15.85 & 8 & $\begin{array}{c}\mathrm{H}, 4-\mathrm{Br}, 2-\mathrm{Cl}, 3-\mathrm{Cl}, 4-\mathrm{Cl}, 4-\mathrm{F}, \\
2-\mathrm{OCH}_{3}, 3-\mathrm{NO}_{2}\end{array}$ \\
\hline & $\sigma^{+}$ & 0.906 & 1136.05 & -29.59 & 14.54 & 8 & $\begin{array}{c}\mathrm{H}, 4-\mathrm{Br}, 2-\mathrm{Cl}, 3-\mathrm{Cl}, 4-\mathrm{Cl}, 4-\mathrm{F}, \\
\text { 2- } \mathrm{OCH}_{3}, 3-\mathrm{NO}_{2},\end{array}$ \\
\hline & $\sigma_{\mathrm{I}}$ & 0.825 & 1141.79 & -30.22 & 18.31 & 9 & $\begin{array}{c}\mathrm{H}, 4-\mathrm{Br}, 2-\mathrm{Cl}, 3-\mathrm{Cl}, 4-\mathrm{Cl}, 4-\mathrm{F}, \\
2-\mathrm{OCH}_{3}, 3-\mathrm{NO}_{2}, 4-\mathrm{NO}_{2}\end{array}$ \\
\hline & $\sigma_{\mathrm{R}}$ & 0.845 & 1122.76 & -32.88 & 17.23 & 9 & $\begin{array}{c}\mathrm{H}, 4-\mathrm{Br}, 2-\mathrm{Cl}, 3-\mathrm{Cl}, 4-\mathrm{Cl}, 4-\mathrm{F}, \\
2-\mathrm{OCH}_{3}, 3-\mathrm{NO}_{2}, 4-\mathrm{NO}_{2}\end{array}$ \\
\hline & $\mathrm{F}$ & 0.845 & 1137.43 & -20.21 & 18.79 & 9 & $\begin{array}{c}\mathrm{H}, 4-\mathrm{Br}, 2-\mathrm{Cl}, 3-\mathrm{Cl}, 4-\mathrm{Cl}, 4-\mathrm{F}, \\
2-\mathrm{OCH}_{3}, 3-\mathrm{NO}_{2}, 4-\mathrm{NO}_{2}\end{array}$ \\
\hline & $\mathrm{R}$ & 0.842 & 1122.74 & -29.19 & 17.59 & 9 & $\begin{array}{c}\mathrm{H}, 4-\mathrm{Br}, 2-\mathrm{Cl}, 3-\mathrm{Cl}, 4-\mathrm{Cl}, 4-\mathrm{F}, \\
2-\mathrm{OCH}_{3}, 3-\mathrm{NO}_{2}, 4-\mathrm{NO}_{2}\end{array}$ \\
\hline \multirow{6}{*}{$\mathrm{CH}_{o p}$} & $\sigma$ & 0.902 & 823.40 & -1.811 & 5.001 & 9 & $\begin{array}{c}\mathrm{H}, 4-\mathrm{Br}, 2-\mathrm{Cl}, 3-\mathrm{Cl}, 4-\mathrm{Cl} \\
\text { 4-F, 2-- } \mathrm{OCH}_{3}, 3-\mathrm{NO}_{2}, 4-\mathrm{NO}_{2}\end{array}$ \\
\hline & $\sigma^{+}$ & 0.905 & 823.36 & -1.553 & 5.004 & 9 & $\begin{array}{l}\mathrm{H}, 4-\mathrm{Br}, 2-\mathrm{Cl}, 3-\mathrm{Cl}, 4-\mathrm{Cl}, \\
4-\mathrm{F}, 2-\mathrm{OCH}_{3}, 3-\mathrm{NO}_{2}, 4-\mathrm{NO}_{2}\end{array}$ \\
\hline & $\sigma_{\mathrm{I}}$ & 0.815 & 824.59 & -3.665 & 4.989 & 9 & $\begin{array}{c}\mathrm{H}, 4-\mathrm{Br}, 2-\mathrm{Cl}, 3-\mathrm{Cl}, 4-\mathrm{Cl}, \\
4-\mathrm{F}, 2-\mathrm{OCH}_{3}, 3-\mathrm{NO}_{2}, 4-\mathrm{NO}_{2}\end{array}$ \\
\hline & $\sigma_{\mathrm{R}}$ & 0.810 & 822.62 & -1.995 & 5.020 & 9 & $\begin{array}{c}\mathrm{H}, 4-\mathrm{Br}, 2-\mathrm{Cl}, 3-\mathrm{Cl}, 4-\mathrm{Cl}, \\
4-\mathrm{F}, 2-\mathrm{OCH}_{3}, 3-\mathrm{NO}_{2}, 4-\mathrm{NO}_{2}\end{array}$ \\
\hline & $\mathrm{F}$ & 0.803 & 823.34 & -0.847 & 5.045 & 9 & $\begin{array}{c}\mathrm{H}, 4-\mathrm{Br}, 2-\mathrm{Cl}, 3-\mathrm{Cl}, 4-\mathrm{Cl}, 4-\mathrm{F} \\
2-\mathrm{OCH}_{3}, 3-\mathrm{NO}_{2}, 4-\mathrm{NO}_{2}\end{array}$ \\
\hline & $\mathrm{R}$ & 0.811 & 822.57 & -1.995 & 5.018 & 9 & $\begin{array}{c}\mathrm{H}, 4-\mathrm{Br}, 2-\mathrm{Cl}, 3-\mathrm{Cl}, 4-\mathrm{Cl} \\
4-\mathrm{F}, 2-\mathrm{OCH}_{3}, 3-\mathrm{NO}_{2}, 4-\mathrm{NO}_{2}\end{array}$ \\
\hline \multirow{2}{*}{$\mathrm{CH}=\mathrm{CH}_{o p}$} & $\sigma$ & 0.903 & 1021.49 & -3.793 & 4.420 & 8 & $\begin{array}{c}\mathrm{H}, 4-\mathrm{Br}, 2-\mathrm{Cl}, 3-\mathrm{Cl}, 4-\mathrm{Cl}, \\
4-\mathrm{F}, 2-\mathrm{OCH}_{3}, 3-\mathrm{NO}_{2},\end{array}$ \\
\hline & $\sigma^{+}$ & 0.903 & 1021.62 & -4.085 & 4.307 & 8 & $\begin{array}{c}\mathrm{H}, 4-\mathrm{Br}, 2-\mathrm{Cl}, 3-\mathrm{Cl}, 4-\mathrm{Cl}, \\
\text { 4-F, 2-- } \mathrm{OCH}_{3}, 3-\mathrm{NO}_{2},\end{array}$ \\
\hline
\end{tabular}




\begin{tabular}{|c|c|c|c|c|c|c|c|}
\hline & $\sigma_{\mathrm{I}}$ & 0.832 & 1023.70 & -7.085 & 4.409 & 9 & $\begin{array}{c}\mathrm{H}, 4-\mathrm{Br}, 2-\mathrm{Cl}, 3-\mathrm{Cl}, 4-\mathrm{Cl}, \\
4-\mathrm{F}, 2-\mathrm{OCH}_{3}, 3-\mathrm{NO}_{2}, 4-\mathrm{NO}_{2}\end{array}$ \\
\hline & $\sigma_{\mathrm{R}}$ & 0.813 & 1020.17 & -2.245 & 4.017 & 9 & $\begin{array}{c}\mathrm{H}, 4-\mathrm{Br}, 2-\mathrm{Cl}, 3-\mathrm{Cl}, 4-\mathrm{Cl}, \\
4-\mathrm{F}, 2-\mathrm{OCH}_{3}, 3-\mathrm{NO}_{2}, 4-\mathrm{NO}_{2}\end{array}$ \\
\hline & $\mathrm{F}$ & 0.819 & 1022.26 & -3.798 & 4.569 & 9 & $\begin{array}{l}\mathrm{H}, 4-\mathrm{Br}, 2-\mathrm{Cl}, 3-\mathrm{Cl}, 4-\mathrm{Cl}, \\
\text { 4-F, 2- } \mathrm{OCH}_{3}, 3-\mathrm{NO}_{2}, 4-\mathrm{NO}_{2}\end{array}$ \\
\hline & $\mathrm{R}$ & 0.815 & 1020.07 & -2.515 & 4.604 & 9 & $\begin{array}{c}\mathrm{H}, 4-\mathrm{Br}, 2-\mathrm{Cl}, 3-\mathrm{Cl}, 4-\mathrm{Cl}, \\
\text { 4-F, 2-- } \mathrm{OCH}_{3}, 3-\mathrm{NO}_{2}, 4-\mathrm{NO}_{2}\end{array}$ \\
\hline \multirow{6}{*}{$\mathrm{C}=\mathrm{C}_{o p}$} & $\sigma$ & 0.812 & 532.98 & 13.14 & 25.56 & 9 & $\begin{array}{c}\mathrm{H}, 4-\mathrm{Br}, 2-\mathrm{Cl}, 3-\mathrm{Cl}, 4-\mathrm{Cl}, \\
4-\mathrm{F}, 2-\mathrm{OCH}_{3}, 3-\mathrm{NO}_{2}, 4-\mathrm{NO}_{2}\end{array}$ \\
\hline & $\sigma^{+}$ & 0.832 & 530.38 & 22.42 & 24.17 & 9 & $\begin{array}{l}\mathrm{H}, 4-\mathrm{Br}, 2-\mathrm{Cl}, 3-\mathrm{Cl}, 4-\mathrm{Cl}, \\
4-\mathrm{F}, 2-\mathrm{OCH}_{3}, 3-\mathrm{NO}_{2}, 4-\mathrm{NO}_{2}\end{array}$ \\
\hline & $\sigma_{\mathrm{I}}$ & 0.828 & 551.83 & -35.15 & 24.97 & 9 & $\begin{array}{c}\mathrm{H}, 4-\mathrm{Br}, 2-\mathrm{Cl}, 3-\mathrm{Cl}, 4-\mathrm{Cl} \\
4-\mathrm{F}, 2-\mathrm{OCH}_{3}, 3-\mathrm{NO}_{2}, 4-\mathrm{NO}_{2}\end{array}$ \\
\hline & $\sigma_{\mathrm{R}}$ & 0.905 & 544.83 & 50.70 & 22.15 & 8 & $\begin{array}{l}\mathrm{H}, 4-\mathrm{Br}, 2-\mathrm{Cl}, 3-\mathrm{Cl}, 4-\mathrm{Cl}, \\
\text { 2- } \mathrm{OCH}_{3}, 3-\mathrm{NO}_{2}, 4-\mathrm{NO}_{2}\end{array}$ \\
\hline & $\mathrm{F}$ & 0.905 & 563.68 & -61.19 & 21.63 & 8 & $\begin{array}{c}\mathrm{H}, 4-\mathrm{Br}, 2-\mathrm{Cl}, 3-\mathrm{Cl}, 4-\mathrm{Cl} \\
2-\mathrm{OCH}_{3}, 3-\mathrm{NO}_{2}, 4-\mathrm{NO}_{2}\end{array}$ \\
\hline & $\mathrm{R}$ & 0.906 & 547.60 & 59.23 & 20.10 & 7 & $\begin{array}{c}\mathrm{H}, 4-\mathrm{Br}, 3-\mathrm{Cl}, 4-\mathrm{Cl}, 2-\mathrm{OCH}_{3}, \\
\text { 3- }-\mathrm{NO}_{2}, 4-\mathrm{NO}_{2}\end{array}$ \\
\hline \multirow{6}{*}{$\delta \mathrm{H} \alpha$} & $\sigma$ & 0.906 & 7.466 & 0.184 & 0.09 & 8 & $\begin{array}{l}\mathrm{H}, 4-\mathrm{Br}, 2-\mathrm{Cl}, 4-\mathrm{Cl}, 4-\mathrm{F} \\
2-\mathrm{OCH}_{3}, 3-\mathrm{NO}_{2}, 4-\mathrm{NO}_{2}\end{array}$ \\
\hline & $\sigma^{+}$ & 0.905 & 7.470 & 0.158 & 0.09 & 8 & $\begin{array}{l}\mathrm{H}, 4-\mathrm{Br}, 2-\mathrm{Cl}, 4-\mathrm{Cl}, 4-\mathrm{F}, \\
2-\mathrm{OCH}_{3}, 3-\mathrm{NO}_{2}, 4-\mathrm{NO}_{2}\end{array}$ \\
\hline & $\sigma_{\mathrm{I}}$ & 0.829 & 7.440 & 0.160 & 0.11 & 9 & $\begin{array}{l}\mathrm{H}, 4-\mathrm{Br}, 2-\mathrm{Cl}, 3-\mathrm{Cl}, 4-\mathrm{Cl}, \\
\text { 4-F, 2- } \mathrm{OCH}_{3}, 3-\mathrm{NO}_{2}, 4-\mathrm{NO}_{2}\end{array}$ \\
\hline & $\sigma_{\mathrm{R}}$ & 0.907 & 7.564 & 0.310 & 0.07 & 8 & $\begin{array}{l}\mathrm{H}, 4-\mathrm{Br}, 2-\mathrm{Cl}, 4-\mathrm{Cl}, 4-\mathrm{F} \\
2-\mathrm{OCH}_{3}, 3-\mathrm{NO}_{2}, 4-\mathrm{NO}_{2}\end{array}$ \\
\hline & $\mathrm{F}$ & 0.822 & 7.460 & 0.114 & 0.11 & 9 & $\begin{array}{l}\mathrm{H}, 4-\mathrm{Br}, 2-\mathrm{Cl}, 3-\mathrm{Cl}, 4-\mathrm{Cl}, \\
4-\mathrm{F}, 2-\mathrm{OCH}_{3}, 3-\mathrm{NO}_{2}, 4-\mathrm{NO}_{2}\end{array}$ \\
\hline & $\mathrm{R}$ & 0.904 & 7.567 & 0.288 & 0.08 & 8 & $\begin{array}{l}\mathrm{H}, 4-\mathrm{Br}, 2-\mathrm{Cl}, 4-\mathrm{Cl}, 4-\mathrm{F} \\
\text { 2- } \mathrm{OCH}_{3}, 3-\mathrm{NO}_{2}, 4-\mathrm{NO}_{2}\end{array}$ \\
\hline \multirow{6}{*}{$\delta \mathrm{H} \beta$} & $\sigma$ & 0.812 & 7.704 & 0.067 & 0.15 & 9 & $\begin{array}{c}\mathrm{H}, 4-\mathrm{Br}, 2-\mathrm{Cl}, 3-\mathrm{Cl}, 4-\mathrm{Cl}, \\
4-\mathrm{F}, 2-\mathrm{OCH}_{3}, 3-\mathrm{NO}_{2}, 4-\mathrm{NO}_{2}\end{array}$ \\
\hline & $\sigma^{+}$ & 0.924 & 7.689 & 0.122 & 0.14 & 9 & $\begin{array}{c}\mathrm{H}, 4-\mathrm{Br}, 2-\mathrm{Cl}, 3-\mathrm{Cl}, 4-\mathrm{Cl}, \\
\text { 4-F, 2-- } \mathrm{OCH}_{3}, 3-\mathrm{NO}_{2}, 4-\mathrm{NO}_{2}\end{array}$ \\
\hline & $\sigma_{\mathrm{I}}$ & 0.812 & 7.681 & 0.088 & 0.15 & 9 & $\begin{array}{c}\mathrm{H}, 4-\mathrm{Br}, 2-\mathrm{Cl}, 3-\mathrm{Cl}, 4-\mathrm{Cl}, \\
4-\mathrm{F}, 2-\mathrm{OCH}_{3}, 3-\mathrm{NO}_{2}, 4-\mathrm{NO}_{2}\end{array}$ \\
\hline & $\sigma_{\mathrm{R}}$ & 0.814 & 7.734 & 0.080 & 0.15 & 9 & $\begin{array}{l}\mathrm{H}, 4-\mathrm{Br}, 2-\mathrm{Cl}, 3-\mathrm{Cl}, 4-\mathrm{Cl} \text {, } \\
\text { 4-F, 2-- } \mathrm{OCH}_{3}, 3-\mathrm{NO}_{2}, 4-\mathrm{NO}_{2}\end{array}$ \\
\hline & $\mathrm{F}$ & 0.801 & 7.716 & 0.010 & 0.15 & 9 & $\begin{array}{c}\mathrm{H}, 4-\mathrm{Br}, 2-\mathrm{Cl}, 3-\mathrm{Cl}, 4-\mathrm{Cl}, \\
\text { 4-F, 2-- } \mathrm{OCH}_{3}, 3-\mathrm{NO}_{2}, 4-\mathrm{NO}_{2}\end{array}$ \\
\hline & $\mathrm{R}$ & 0.821 & 7.743 & 0.114 & 0.14 & 9 & $\begin{array}{l}\mathrm{H}, 4-\mathrm{Br}, 2-\mathrm{Cl}, 3-\mathrm{Cl}, 4-\mathrm{Cl}, \\
4-\mathrm{F}, 2-\mathrm{OCH}_{3}, 3-\mathrm{NO}_{2}, 4-\mathrm{NO}_{2}\end{array}$ \\
\hline \multirow{5}{*}{$\delta \mathrm{CO}$} & $\sigma$ & 0.997 & 190.36 & -1.271 & 0.110 & 9 & $\begin{array}{c}\mathrm{H}, 4-\mathrm{Br}, 2-\mathrm{Cl}, 3-\mathrm{Cl}, 4-\mathrm{Cl}, \\
4-\mathrm{F}, 2-\mathrm{OCH}_{3}, 3-\mathrm{NO}_{2}, 4-\mathrm{NO}_{2}\end{array}$ \\
\hline & $\sigma^{+}$ & 0.995 & 190.32 & -1.042 & 0.223 & 9 & $\begin{array}{l}\mathrm{H}, 4-\mathrm{Br}, 2-\mathrm{Cl}, 3-\mathrm{Cl}, 4-\mathrm{Cl}, \\
4-\mathrm{F}, 2-\mathrm{OCH}_{3}, 3-\mathrm{NO}_{2}, 4-\mathrm{NO}_{2}\end{array}$ \\
\hline & $\sigma_{\mathrm{I}}$ & 0.907 & 190.86 & -1.835 & 0.321 & 9 & $\begin{array}{l}\mathrm{H}, 4-\mathrm{Br}, 2-\mathrm{Cl}, 3-\mathrm{Cl}, 4-\mathrm{Cl}, \\
4-\mathrm{F}, 2-\mathrm{OCH}_{3}, 3-\mathrm{NO}_{2}, 4-\mathrm{NO}_{2}\end{array}$ \\
\hline & $\sigma_{\mathrm{R}}$ & 0.908 & 189.80 & -1.424 & 0.325 & 8 & $\begin{array}{c}\text { 4- } \mathrm{Br}, 2-\mathrm{Cl}, 3-\mathrm{Cl}, 4-\mathrm{Cl}, 4-\mathrm{F} \\
2-\mathrm{OCH}_{3}, 3-\mathrm{NO}_{2}, 4-\mathrm{NO}_{2}\end{array}$ \\
\hline & $\mathrm{F}$ & 0.906 & 190.67 & -1.394 & 0.380 & 7 & $\begin{array}{c}\mathrm{H}, 4-\mathrm{Br}, 2-\mathrm{Cl}, 3-\mathrm{Cl}, 4-\mathrm{Cl}, \\
\text { 3- } \mathrm{NO}_{2}, 4-\mathrm{NO}_{2}\end{array}$ \\
\hline
\end{tabular}




\begin{tabular}{|c|c|c|c|c|c|c|c|}
\hline & $\mathrm{R}$ & 0.907 & 189.80 & -1.285 & 0.353 & 8 & $\begin{array}{c}\text { 4- } \mathrm{Br}, 2-\mathrm{Cl}, 3-\mathrm{Cl}, 4-\mathrm{Cl}, 4-\mathrm{F}, \\
\text { 2- } \mathrm{OCH}_{3}, 3-\mathrm{NO}_{2}, 4-\mathrm{NO}_{2}\end{array}$ \\
\hline \multirow{6}{*}{$\delta \mathrm{C} \alpha$} & $\sigma$ & 0.907 & 122.86 & -2.073 & 0.757 & 8 & $\begin{array}{c}\mathrm{H}, 4-\mathrm{Br}, 2-\mathrm{Cl}, 3-\mathrm{Cl}, 4-\mathrm{Cl}, \\
4-\mathrm{F}, 3-\mathrm{NO}_{2}, 4-\mathrm{NO}_{2}\end{array}$ \\
\hline & $\sigma^{+}$ & 0.907 & 122.84 & -1.903 & 0.731 & 8 & $\begin{array}{c}\mathrm{H}, 4-\mathrm{Br}, 2-\mathrm{Cl}, 3-\mathrm{Cl}, 4-\mathrm{Cl}, \\
4-\mathrm{F}, 3-\mathrm{NO}_{2}, 4-\mathrm{NO}_{2}\end{array}$ \\
\hline & $\sigma_{\mathrm{I}}$ & 0.824 & 123.18 & -1.863 & 1.030 & 9 & $\begin{array}{c}\mathrm{H}, 4-\mathrm{Br}, 2-\mathrm{Cl}, 3-\mathrm{Cl}, 4-\mathrm{Cl} \\
4-\mathrm{F}, 2-\mathrm{OCH}_{3}, 3-\mathrm{NO}_{2}, 4-\mathrm{NO}_{2}\end{array}$ \\
\hline & $\sigma_{\mathrm{R}}$ & 0.906 & 121.90 & -2.635 & 0.841 & 8 & $\begin{array}{c}\mathrm{H}, 4-\mathrm{Br}, 2-\mathrm{Cl}, 3-\mathrm{Cl}, 4-\mathrm{Cl} \\
\text { 4-F, 3- } \mathrm{NO}_{2}, 4-\mathrm{NO}_{2}\end{array}$ \\
\hline & $\mathrm{F}$ & 0.821 & 123.03 & -1.519 & 1.042 & 9 & $\begin{array}{c}\mathrm{H}, 4-\mathrm{Br}, 2-\mathrm{Cl}, 3-\mathrm{Cl}, 4-\mathrm{Cl} \\
4-\mathrm{F}, 2-\mathrm{OCH}_{3}, 3-\mathrm{NO}_{2}, 4-\mathrm{NO}_{2}\end{array}$ \\
\hline & $\mathrm{R}$ & 0.854 & 121.93 & -2.198 & 0.915 & 9 & $\begin{array}{c}\mathrm{H}, 4-\mathrm{Br}, 2-\mathrm{Cl}, 3-\mathrm{Cl}, 4-\mathrm{Cl} \\
4-\mathrm{F}, 2-\mathrm{OCH}_{3}, 3-\mathrm{NO}_{2}, 4-\mathrm{NO}_{2}\end{array}$ \\
\hline \multirow{6}{*}{$\delta \mathrm{C} \beta$} & $\sigma$ & 0.901 & 136.38 & 1.698 & 3.719 & 8 & $\begin{array}{c}\mathrm{H}, 4-\mathrm{Br}, 2-\mathrm{Cl}, 3-\mathrm{Cl}, 4-\mathrm{Cl}, \\
4-\mathrm{F}, 3-\mathrm{NO}_{2}, 4-\mathrm{NO}_{2}\end{array}$ \\
\hline & $\sigma^{+}$ & 0.901 & 136.61 & 0.745 & 3.763 & 8 & $\begin{array}{c}\mathrm{H}, 4-\mathrm{Br}, 2-\mathrm{Cl}, 3-\mathrm{Cl}, 4-\mathrm{Cl}, \\
4-\mathrm{F}, 3-\mathrm{NO}_{2}, 4-\mathrm{NO}_{2}\end{array}$ \\
\hline & $\sigma_{\mathrm{I}}$ & 0.801 & 135.97 & 1.863 & 3.756 & 9 & $\begin{array}{c}\mathrm{H}, 4-\mathrm{Br}, 2-\mathrm{Cl}, 3-\mathrm{Cl}, 4-\mathrm{Cl} \\
4-\mathrm{F}, 2-\mathrm{OCH}_{3}, 3-\mathrm{NO}_{2}, 4-\mathrm{NO}_{2}\end{array}$ \\
\hline & $\sigma_{\mathrm{R}}$ & 0.821 & 137.30 & 2.923 & 3.693 & 9 & $\begin{array}{c}\mathrm{H}, 4-\mathrm{Br}, 2-\mathrm{Cl}, 3-\mathrm{Cl}, 4-\mathrm{Cl}, \\
4-\mathrm{F}, 2-\mathrm{OCH}_{3}, 3-\mathrm{NO}_{2}, 4-\mathrm{NO}_{2}\end{array}$ \\
\hline & $\mathrm{F}$ & 0.804 & 137.10 & -0.670 & 3.774 & 9 & $\begin{array}{c}\mathrm{H}, 4-\mathrm{Br}, 2-\mathrm{Cl}, 3-\mathrm{Cl}, 4-\mathrm{Cl} \\
4-\mathrm{F}, 2-\mathrm{OCH}_{3}, 3-\mathrm{NO}_{2}, 4-\mathrm{NO}_{2}\end{array}$ \\
\hline & $\mathrm{R}$ & 0.903 & 137.59 & 4.076 & 3.601 & 8 & $\begin{array}{c}\mathrm{H}, 4-\mathrm{Br}, 2-\mathrm{Cl}, 3-\mathrm{Cl}, 4-\mathrm{Cl}, \\
4-\mathrm{F}, 3-\mathrm{NO}_{2}, 4-\mathrm{NO}_{2}\end{array}$ \\
\hline
\end{tabular}

\section{2. IR spectral study}

The synthesized chalcones in the present study are shown in Scheme 1. The carbonyl stretching frequencies $\left(\mathrm{cm}^{-1}\right)$ of s-cis and s-trans of isomers are tabulated in Table 2 and the corresponding conformers are shown in Fig. 2.<smiles>[X]c1cccc(C=CC(=O)c2ccc(OC)cc2OC)c1</smiles><smiles>[X]c1cccc(/C=C/C(=O)c2ccc(OC)cc2OC)c1</smiles>

Fig. 2. The $s$-cis and $s$-trans conformers of 2,4-dimethoxy phenyl chalcones.

The stretching frequencies for carbonyl absorption are assigned based on the assignments made by Hays and Timmons [2] for $s$-cis and s-trans conformers at 1690 and $1670 \mathrm{~cm}^{-1}$, respectively. The assigned infrared COs-cis and s-trans conformers have been correlated with Hammett substituent constants and Swain-Lupton constants [37]. In this correlation the structure parameter Hammett equation employed is as shown in the following equation:

$$
v=\rho \sigma+v_{0}
$$


where $v$ is the carbonyl frequencies of substituted system and $v_{0}$ is the corresponding quantity of unsubstitued system; $\sigma$ is a Hammett substituent constant, which in principle is characteristics of the substituent and $\rho$ is a reaction constant which is depend upon the nature of the reaction.

The results of statistical analysis [1,2,6,10,19,21,23-25,28,37] were shown in Table 3, From Table 3, The resonance component only correlated satisfactorily with $\mathrm{CO}$ s-cis conformers stretches. The remaining Hammett substituent constants and $\mathrm{F}$ parameters were failing in correlation. The Hammett $\sigma_{\mathrm{I}}$ and $\mathrm{F}$ parameters gave satisfactory correlation with $\mathrm{CO}$ $s$-trans conformers stretches. The remaining Hammett substituent constants and R parameters were failing in correlation. This is due to the reasons stated in earlier and associated with the resonance conjugative structure shown in Fig. 1.

The correlation of $\mathrm{CH}_{i p}$ modes with Hammett $\sigma$ and $\sigma^{+}$constant gave satisfactory correlation. The remaining Hammett substituent constants, $\mathrm{F}$ and $\mathrm{R}$ parameters were failing in correlation. The Hammett $\sigma$ and $\sigma^{+}$constants correlated satisfactorily with $\mathrm{CH}_{o p}$ modes of all chalcones. The remaining Hammett substituent constants, $\mathrm{F}$ and $\mathrm{R}$ parameters gave poor correlation. This failure in correlation is due to the incapability of polar and inductive effects of the substituents and associated with the resonance conjugative structure as shown in Fig. 1.

The Hammett $\sigma$ and $\sigma^{+}$constants gave satisfactory correlation with $\mathrm{CH}=\mathrm{CH}_{o p}$ modes.

The remaining Hammett substituent constants and $\mathrm{F}$ and $\mathrm{R}$ parameter failed in correlation due to the inductive, resonance and field components of the substituents. The Hammett $\sigma_{R}$ constants, $\mathrm{F}$ and $\mathrm{R}$ were satisfactorily correlated with $\mathrm{C}=\mathrm{C}_{o p}$ modes. The inductive components of the substituents $\sigma, \sigma^{+}$and $\sigma_{\mathrm{I}}$ constants failed in correlation. This is due to the reasons stated earlier and associated with the resonance conjugative structure as shown in Fig. 1. Some of the single parameter correlations with Hammett substituent constants were not obeyed in the regression. While seeking these parameters in multi-regression, with $\mathrm{F}$ and $\mathrm{R}$ Swain-Lupton's constants [37], they gave satisfactory correlations with the infrared red group frequencies. The multi correlation equations are given in (5-16).

$$
\begin{aligned}
& v \mathrm{CO}_{\text {s-cis }}\left(\mathrm{cm}^{-1}\right)=1615.14( \pm 28.913)+29.85( \pm 55.27) \sigma_{\mathrm{I}}+31.83( \pm 43.226) \sigma_{\mathrm{R}} \\
& (R=0.938, \mathrm{n}=9, \mathrm{P}>90 \%) \\
& v \mathrm{CO}_{\text {s-cis }}\left(\mathrm{cm}^{-1}\right)=1618.40( \pm 25.419)+23.55( \pm 49.20) \mathrm{F}+31.11( \pm 41.755) \mathrm{R} \\
& (R=0.933, \mathrm{n}=9, \mathrm{P}>90 \%) \\
& v \mathrm{CO}_{s-\text { trans }}\left(\mathrm{cm}^{-1}\right)=1585.78( \pm 36.70)-70.10( \pm 70.17) \sigma_{\mathrm{I}}+27.97( \pm 54.873) \sigma_{\mathrm{R}} \\
& (R=0.939, \mathrm{n}=9, \mathrm{P}>90 \%) \\
& v \mathrm{CO}_{\text {s-trans }}\left(\mathrm{cm}^{-1}\right)=1572.50( \pm 32.75)-48.63( \pm 63.40) \mathrm{F}+4.185( \pm 53.804) \mathrm{R} \\
& (R=0.930, \mathrm{n}=9, \mathrm{P}>90 \%) \\
& v \mathrm{CH}_{i p}\left(\mathrm{~cm}^{-1}\right)=1133.31( \pm 17.133)-22.305( \pm 32.757) \sigma_{\mathrm{I}}-29.183( \pm 25.615) \sigma_{\mathrm{R}} \\
& (R=0.951, \mathrm{n}=9, \mathrm{P}>95 \%) \\
& v \mathrm{CH}_{i p}\left(\mathrm{~cm}^{-1}\right)=1132.24( \pm 14.962)-21.422( \pm 28.962) \mathrm{F}-29.822( \pm 24.577) \mathrm{R} \\
& (R=0.949, \mathrm{n}=9, \mathrm{P}>90 \%) \\
& v \mathrm{CH}_{o p}\left(\mathrm{~cm}^{-1}\right)=824.16( \pm 5.1344)-3.2715( \pm 9.8163) \sigma_{\mathrm{I}}-1.452( \pm 7.6762) \sigma_{\mathrm{R}} \\
& (R=0.917, \mathrm{n}=9, \mathrm{P}>90 \%)
\end{aligned}
$$




$$
\begin{aligned}
& v \mathrm{CH}_{o p}\left(\mathrm{~cm}^{-1}\right)=822.99( \pm 4.4550)-0.9296( \pm 8.6235) \mathrm{F}-2.0228( \pm 7.3179) \mathrm{R} \\
& (R=0.911, \mathrm{n}=9, \mathrm{P}>90 \%) \\
& v \mathrm{CH}=\mathrm{CH}_{o p}\left(\mathrm{~cm}^{-1}\right)=1023.38( \pm 4.540)-6.781( \pm 8.680) \sigma_{\mathrm{I}}-1.119( \pm 6.7882) \sigma_{\mathrm{R}} \\
& (R=0.932, \mathrm{n}=9, \mathrm{P}>90 \%) \\
& v \mathrm{CH}=\mathrm{CH}_{o p}\left(\mathrm{~cm}^{-1}\right)=1021.80( \pm 4.007)-3.904( \pm 7.756) \mathrm{F}-2.629( \pm 6.5823) \mathrm{R} \\
& (R=0.924, \mathrm{n}=9, \mathrm{P}>90 \%) \\
& { } \mathrm{C}=\mathrm{C}_{o p}\left(\mathrm{~cm}^{-1}\right)=569.04( \pm 20.073)-51.226( \pm 38.378) \sigma_{\mathrm{I}}+59.210( \pm 30.011) \sigma_{\mathrm{R}} \\
& (R=0.966, \mathrm{n}=9, \mathrm{P}>95 \%) \\
& v \mathrm{C}=\mathrm{C}_{o p}\left(\mathrm{~cm}^{-1}\right)=573.69( \pm 12.846)-58.862( \pm 24.867) \mathrm{F}+57.517( \pm 21.102) \mathrm{R} \\
& (R=0.983, \mathrm{n}=9, \mathrm{P}>95 \%)
\end{aligned}
$$

\section{3. NMR Spectral study}

\section{3. 1. ${ }^{1} \mathrm{H}$ NMR spectra}

From the ${ }^{1} \mathrm{H}$ NMR spectra of chalcones the chemical shifts $(\delta, \mathrm{ppm}) \mathrm{H} \alpha$ and $\mathrm{H} \beta$ are assigned and tabulated in Table 2. These chemical shifts were correlated with Hammett substituent constants, $\mathrm{F}$ and $\mathrm{R}$ parameters. The statistical analysis [1,2,6,10,19,21,23$25,28,37]$ of these chemical shifts is presented in Table 3 . From Table 3, the H $\alpha$ chemical shifts $(\delta, \mathrm{ppm})$ correlated with Hammett $\sigma, \sigma^{+}, \sigma_{\mathrm{R}}$ constants and R parameters were gave satisfactory correlations with $\mathrm{H} \alpha$ chemical shifts of chalcones.

The remaining Hammett substituent constant $\sigma_{\mathrm{I}}$ and $\mathrm{F}$ parameter were failing in correlations due to the inductive and field effects of the substituents. Hammett $\sigma+$ constant only one gave satisfactory correlation with $\mathrm{H}_{\beta}$ chemical shifts $(\delta$, ppm). The remaining Hammett substituent constants and parameter failure in correlation for both chemical shifts are the reasons stated earlier and associated with the resonance - conjugated structure shown in Fig. 1.

Some of the single parameter correlations with Hammett substituent constants were not obeyed in the regression. While seeking these parameters in multi-regression, with $\mathrm{F}$ and R Swain-Lupton's [37] constants, they gave satisfactory correlations with the chemical shifts of $(\delta, \mathrm{ppm}) \mathrm{H} \alpha$ and $\mathrm{H} \beta$. The multi correlation equations are given in (17-20).

$$
\begin{gathered}
\delta \mathrm{H}_{\alpha}(\mathrm{ppm})=7.526( \pm 0.079)+0.079( \pm 0.152) \sigma_{\mathrm{I}}+0.2969( \pm 0.119) \sigma_{\mathrm{R}} \\
(R=0.974, \mathrm{n}=9, \mathrm{P}>95 \%) \\
\delta \mathrm{H}_{\alpha}(\mathrm{ppm})=7.511( \pm 0.068)+0.126( \pm 0.131) \mathrm{F}+0.292( \pm 0.111) \mathrm{R} \\
(R=0.974, \mathrm{n}=9, \mathrm{P}>95 \%) \\
\delta \mathrm{H}_{\beta}(\mathrm{ppm})=7.701( \pm 0.155)+0.070( \pm 0.297) \sigma_{\mathrm{I}}+0.068( \pm 0.232) \sigma_{\mathrm{R}} \\
(R=0.901, \mathrm{n}=9, \mathrm{P}>90 \%) \\
\delta \mathrm{H}_{\beta}(\mathrm{ppm})=7.736( \pm 0.132)+0.015( \pm 0.256) \mathrm{F}+0.115( \pm 0.217) \mathrm{R} \\
(R=0.902, \mathrm{n}=9, \mathrm{P}>90 \%)
\end{gathered}
$$




\section{3. $2 .{ }^{13} \mathrm{C}$ NMR spectra}

The carbonyl carbon, $C \alpha$ and $C \beta$ chemical shifts $(\delta$, ppm) of 2,4-dimethoxy phenyl chalcones were assigned and tabulated in Table 2. These chemical shifts are correlated [1,2,6,10,19,21,23-25,28,37] with Hammett substituent constants, F and R parameters. The results of statistical analysis are shown in Table 3. From Table 3, the Hammett $\sigma, \sigma^{+}, \sigma_{\mathrm{I}}, \sigma_{\mathrm{R}}$ constants, $\mathrm{F}$ and $\mathrm{R}$ parameters were gave satisfactory correlations with carbonyl carbon chemical shifts of chalcones. The Hammett $\sigma, \sigma^{+}$and $\sigma_{R}$ constants correlated satisfactorily with $C \alpha$ chemical shifts. The $C \beta$ chemical shifts $(\delta$, ppm) were satisfactorily correlated with the Hammett $\sigma, \sigma^{+}$and $\sigma_{\mathrm{R}}$ constants. The remaining Hammett substituent constant $\sigma_{\mathrm{I}}, \mathrm{F}$ and $\mathrm{R}$ parameters were failing in correlations. This is due to the reasons stated in earlier and associated with the resonance conjugative structure shown in Fig. 1.

The multi regression analysis of carbonyl carbon, $C \alpha$ and $C \beta$ chemical shifts $(\delta$, ppm) of 2,4-dimethoxy phenyl chalcones were satisfactorily correlated with $\sigma_{\mathrm{I}}, \sigma_{\mathrm{R}}$ and SwainLuptons' [37] F and R parameters. The multi-regression equation is given in (21-26).

$$
\begin{gathered}
\delta \mathrm{CO}(\mathrm{ppm})=190.52( \pm 0.087)-1.517( \pm 0.167) \sigma_{\mathrm{I}}-1.172( \pm 0.130) \sigma_{\mathrm{R}} \\
(R=0.998, \mathrm{n}=9, \mathrm{P}>95 \%) \\
\delta \mathrm{CO}(\mathrm{ppm})=190.44( \pm 0.072)-1.448( \pm 0.140) \mathrm{F}-1.328( \pm 0.119) \mathrm{R} \\
(R=0.998, \mathrm{n}=9, \mathrm{P}>95 \%) \\
\delta \mathrm{C}_{\alpha}(\mathrm{ppm})=122.47( \pm 0.829)-1.201( \pm 1.585) \sigma_{\mathrm{I}}-2.435( \pm 1.240) \sigma_{\mathrm{R}} \\
(R=0.968, \mathrm{n}=9, \mathrm{P}>95 \%) \\
\delta \mathrm{C}_{\alpha}(\mathrm{ppm})=122.64( \pm 0.739)-1.610( \pm 1.430) \mathrm{F}-2.245( \pm 1.214) \mathrm{R} \\
(R=0.965, \mathrm{n}=9, \mathrm{P}>95 \%) \\
\delta \mathrm{C}_{\beta}(\mathrm{ppm})=136.77( \pm 3.804)+1.120( \pm 7.273) \sigma_{\mathrm{I}}+2.737( \pm 5.687) \sigma_{\mathrm{R}} \\
(R=0.921, \mathrm{n}=9, \mathrm{P}>90 \%) \\
\delta \mathrm{C}_{\beta}(\mathrm{ppm})=137.81( \pm 3.197)-0.505( \pm 6.189) \mathrm{F}+4.061( \pm 5.252) \mathrm{R} \\
(R=0.933, \mathrm{n}=9, \mathrm{P}>90 \%)
\end{gathered}
$$

\section{4. Antimicrobial activities}

Chalcones possess various biological activities such as antibacterial [26,38], antifungal [26], antioxidant [8], antiviral [9], antimalarial [10], antiplasmodial [28], antituberclosis [12], antiproliferative [13], antileshmanial [14], anti-inflammatory [15], antianalgesic and sedative [16], insect antifeedants [13,22]. Halogenated chalcones possess insect antifeedant activities $[13,22,39-44]$. The presence of a reactive $\alpha, \beta$-unsaturated keto function in chalcones was found to be responsible for their antimicrobial activity. At present, scientists have paid more interest to correlate the group frequencies of spectral data with Hammett substituent constants to explain the substituent effects of organic compounds. 


\section{4. 1. Antibacterial sensitivity assay}

Antibacterial sensitivity assay was performed using Kirby-Bauer [30] disc diffusion technique. In each Petri plate about $0.5 \mathrm{~mL}$ of the test bacterial sample was spread uniformly over the solidified Mueller Hinton agar using sterile glass spreader. Then the discs with $5 \mathrm{~mm}$ diameter made up of Whatmann No.1 filter paper, impregnated with the solution of the compound was placed on the medium using sterile foreceps.

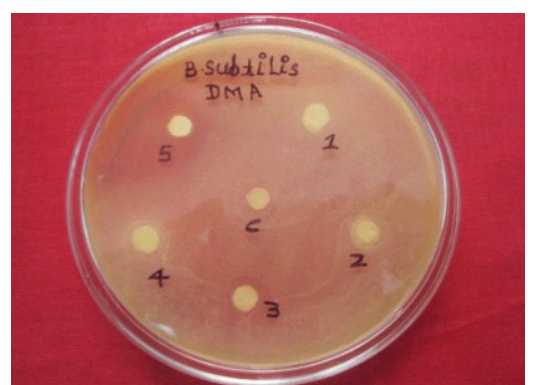

Plate-1

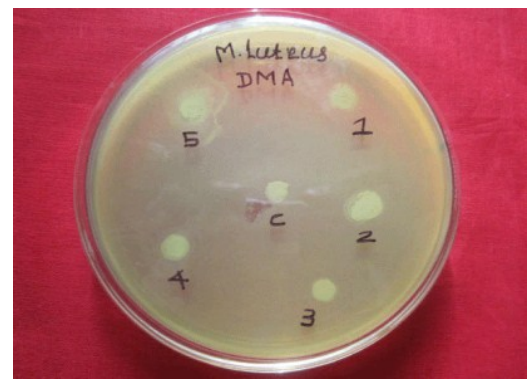

Plate-3

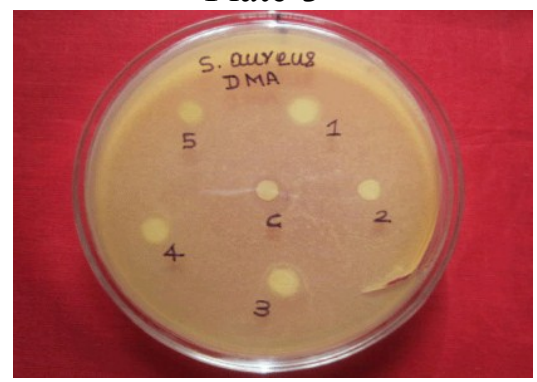

Plate-5

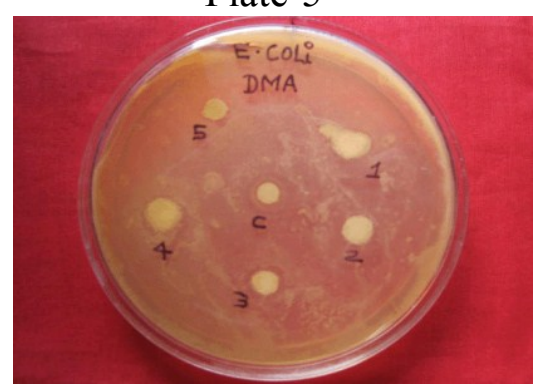

Plate-7

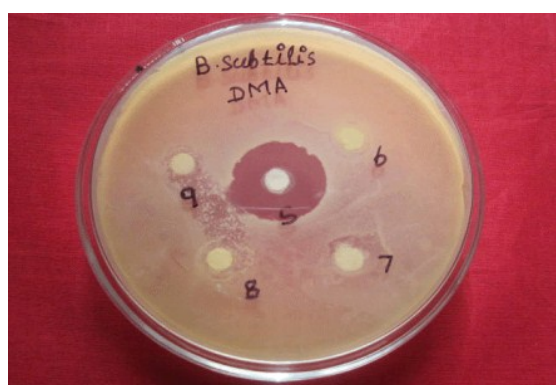

Plate-2

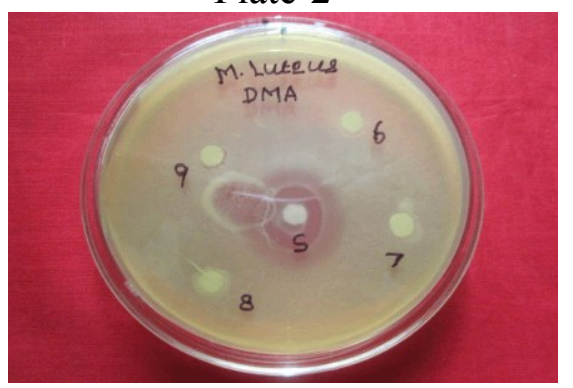

Plate-4

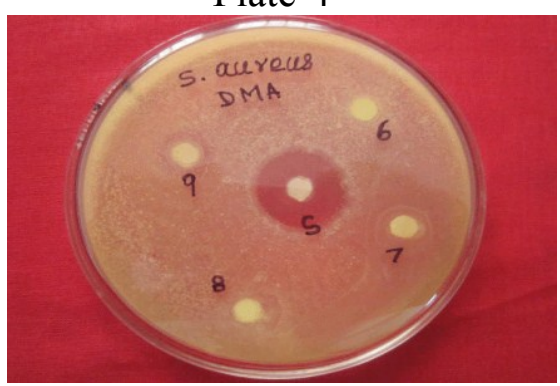

Plate-6

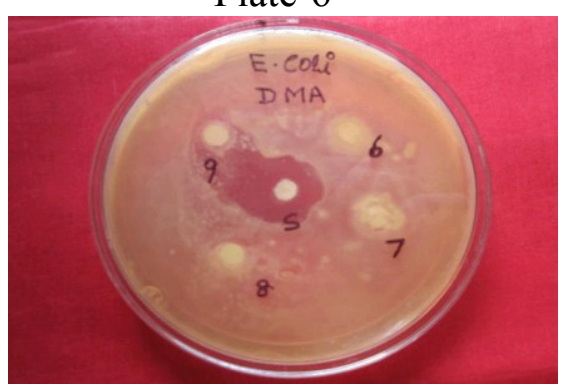

Plate- 8 


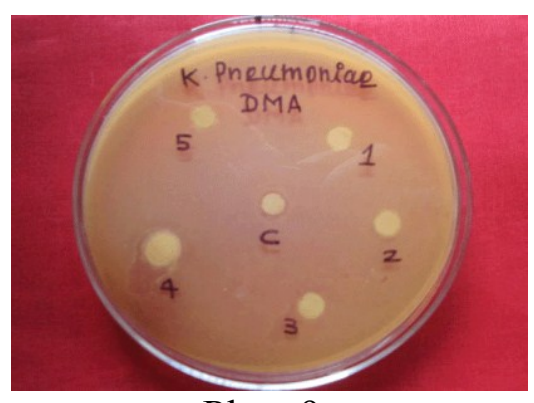

Plate-9

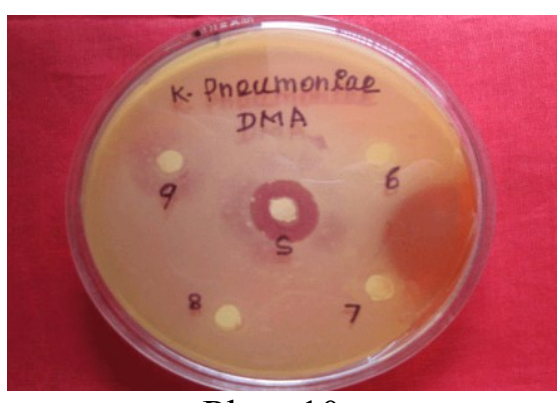

Plate-10

Fig. 3. Antibacterial activities of substituted 2,4-dimethoxy phenyl ketones.

The plates were incubated for 24 hours at $37^{\circ} \mathrm{C}$ by keeping the plates upside down to prevent the collection of water droplets over the medium. After 24 hours, the plates were visually examined and the diameter values of the zone of inhibition were measured. Triplicate results were recorded by repeating the same procedure.

The antibacterial effect of the styryl 2,4-dimethoxy phenyl ketones is shown in Fig. 3 for Plates (1)-(10). Analysis of the zone of inhibition as given Table 4 and the Clustered column Chart Fig. 4, reveals that all the substituents except 4- $\mathrm{NO}_{2}$ and 2-Cl have shown moderate antibacterial activity against all the bacterial species, under investigation.

Table 4. Antibacterial activity of substituted styryl 2,4-dimethoxy phenyl ketones.

\begin{tabular}{|c|c|c|c|c|c|c|}
\hline \multirow{2}{*}{ Entry } & \multirow{2}{*}{ Substt. } & \multicolumn{5}{|c|}{ Zone of Inhibition (mm) } \\
\cline { 3 - 7 } & & \multicolumn{2}{|c|}{ Gram positive Bacteria } & \multicolumn{2}{c|}{ Gram negative Bacteria } \\
\cline { 3 - 7 } & & B. subtilis & M. luteus & S. aureus & E. coli & K. pneumoniae \\
\hline 1 & $\mathrm{H}$ & - & 6 & 6 & 7 & - \\
\hline 2 & $4-\mathrm{Br}$ & 8 & 7 & - & 6 & 6 \\
\hline 3 & $2-\mathrm{Cl}$ & 9 & - & 7 & 8 & 6 \\
\hline 4 & $3-\mathrm{Cl}$ & 8 & - & 6 & 7 & 7 \\
\hline 5 & $4-\mathrm{Cl}$ & - & 9 & - & 6 & - \\
\hline 6 & $4-\mathrm{F}$ & 7 & 6 & - & 6 & - \\
\hline 7 & $2-\mathrm{OCH}_{3}$ & 8 & - & 9 & 6 & 6 \\
\hline 8 & $3-\mathrm{NO}_{2}$ & 8 & 7 & 8 & 7 & 7 \\
\hline 9 & $4-\mathrm{NO}_{2}$ & 7 & 6 & 9 & 9 & 7 \\
\hline Standard & Ampicillin & 14 & 12 & 14 & 13 & 12 \\
\hline Control & $\mathrm{DMSO}^{*}$ & - & - & - & - & - \\
\hline
\end{tabular}




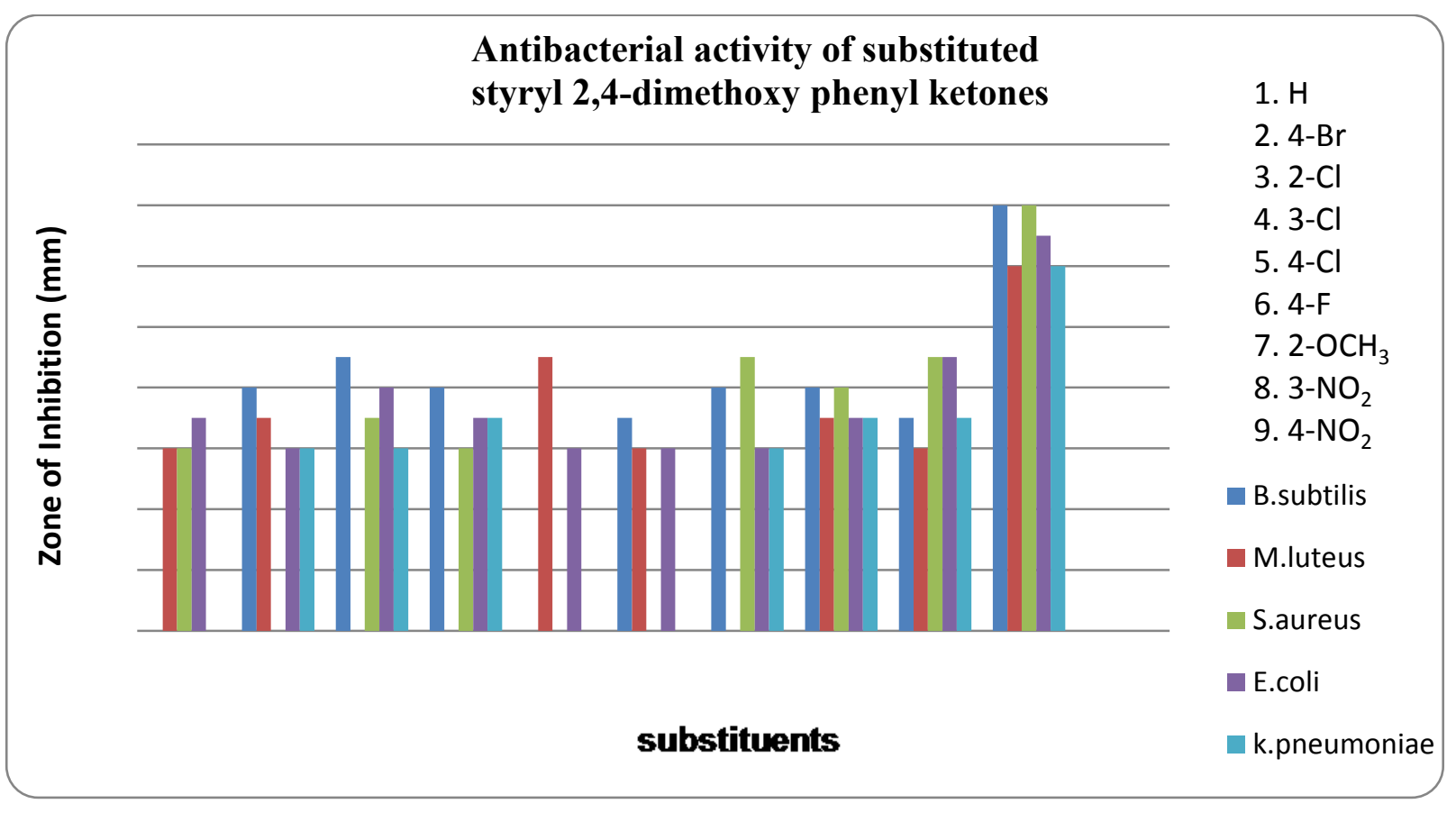

Fig. 4. Antibacterial activity of substituted styryl 2,4-dimethoxy phenyl ketone.

The substituent 4- $\mathrm{NO}_{2}$ has shown more antibacterial activity against $S$. aureus and $E$. coli species. The parent $(\mathrm{H})$ compound has shown moderate antibacterial activity than standard (Ampicillin) against $M$. luteus and $S$. aureus species. The substituent 4-Cl has shown poor activity $M$. luteus and $E$. coli species. The substituent $3-\mathrm{NO}_{2}$ has shown equal activities against all bacteria except $B$. subtilis and $S$. aureus. The compound with 4- $\mathrm{Br}, 3-\mathrm{Cl}, 2-\mathrm{OCH}_{3}$ and $3-\mathrm{NO}_{2}$ substituents has shown equal activities against $B$. subtilis bacterial species. The compound with 2-OCH $\mathrm{OCH}_{3}$ and $3-\mathrm{NO}_{2}$ substituents has shown equal activities against $E$. coli and $K$. pneumoniae bacterial species.

\section{4. 2. Antifungal sensitivity assay}

Antifungal sensitivity assay was performed using Kirby-Bauer [30] disc diffusion technique. PDA medium was prepared and sterilized as above. It was poured (ear bearing heating condition) in the Petri-plate which was already filled with $1 \mathrm{ml}$ of the fungal species. The plate was rotated clockwise and counter clock-wise for uniform spreading of the species. The discs were impregnated with the test solution. The test solution was prepared by dissolving $15 \mathrm{mg}$ of the Chalcone in $1 \mathrm{~mL}$ of DMSO solvent. The medium was allowed to solidify and kept for 24 hours. Then the plates were visually examined and the diameter values of zone of inhibition were measured. Triplicate results were recorded by repeating the same procedure.

The antifungal effect of the styryl 2,4-dimethoxy phenyl ketones is shown in Fig. 5 for Plates (1-4). Analysis of the zone of inhibition as given Table 5 and the Clustered column Chart Fig 6. The substituent 2-Cl and $3-\mathrm{NO}_{2}$ has shown more antifungal activities against all the fungal species namely $A$. niger and $T$. viride. The parent $(\mathrm{H})$ compound and 4-Br substituents have shown moderate antifungal activities against all the fungal species namely A. niger and T. viride, under investigation. The 4-Cl, $4-\mathrm{F}$ and $2-\mathrm{OCH}_{3}$ substituents of this 
have shown inactive for the above two fungal species. The parent compound $(\mathrm{H}), 2-\mathrm{Cl}, 4-\mathrm{Cl}$ and 4-F substituted compound has shown equal activity against fungal species $T$. viride.

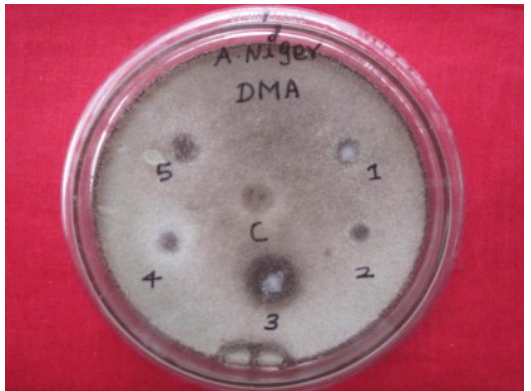

Plate-1

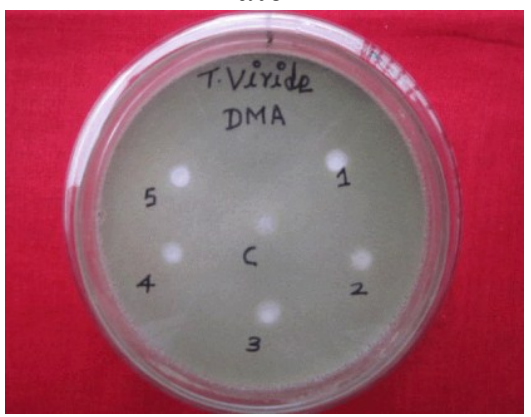

Plate-3

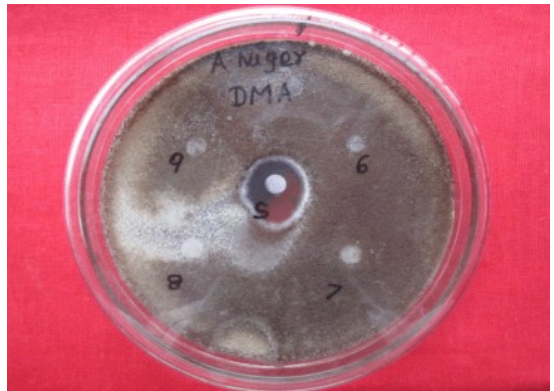

Plate-2

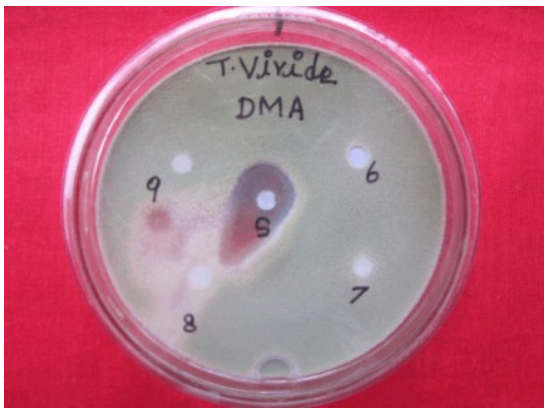

Plate-4

Fig. 5. Antifungal activities substituted styryl 2,4-dimethoxy phenyl ketones.

Table 5. Antifungal activity of substituted styryl 2,4-dimethoxy phenyl ketones.

\begin{tabular}{|c|c|c|c|}
\hline \multirow{2}{*}{ Entry } & \multirow{2}{*}{ Substt. } & \multicolumn{2}{c|}{ Zone of Inhibition (mm) } \\
\cline { 3 - 4 } & & A. niger & T. viride \\
\hline 1 & $\mathrm{H}$ & 7 & 6 \\
\hline 2 & $4-\mathrm{Br}$ & 6 & - \\
\hline 3 & $2-\mathrm{Cl}$ & 9 & 6 \\
\hline 4 & $3-\mathrm{Cl}$ & 6 & - \\
\hline 5 & $4-\mathrm{Cl}$ & - & 6 \\
\hline 6 & $4-\mathrm{F}$ & - & 6 \\
\hline 7 & $2-\mathrm{OCH}_{3}$ & - & - \\
\hline 8 & $3-\mathrm{NO}_{2}$ & 7 & 8 \\
\hline 9 & $4-\mathrm{NO}_{2}$ & 6 & - \\
\hline Standard & Miconazole & 12 & 13 \\
\hline control & $\mathrm{DMSO}^{2}$ & - & - \\
\hline
\end{tabular}




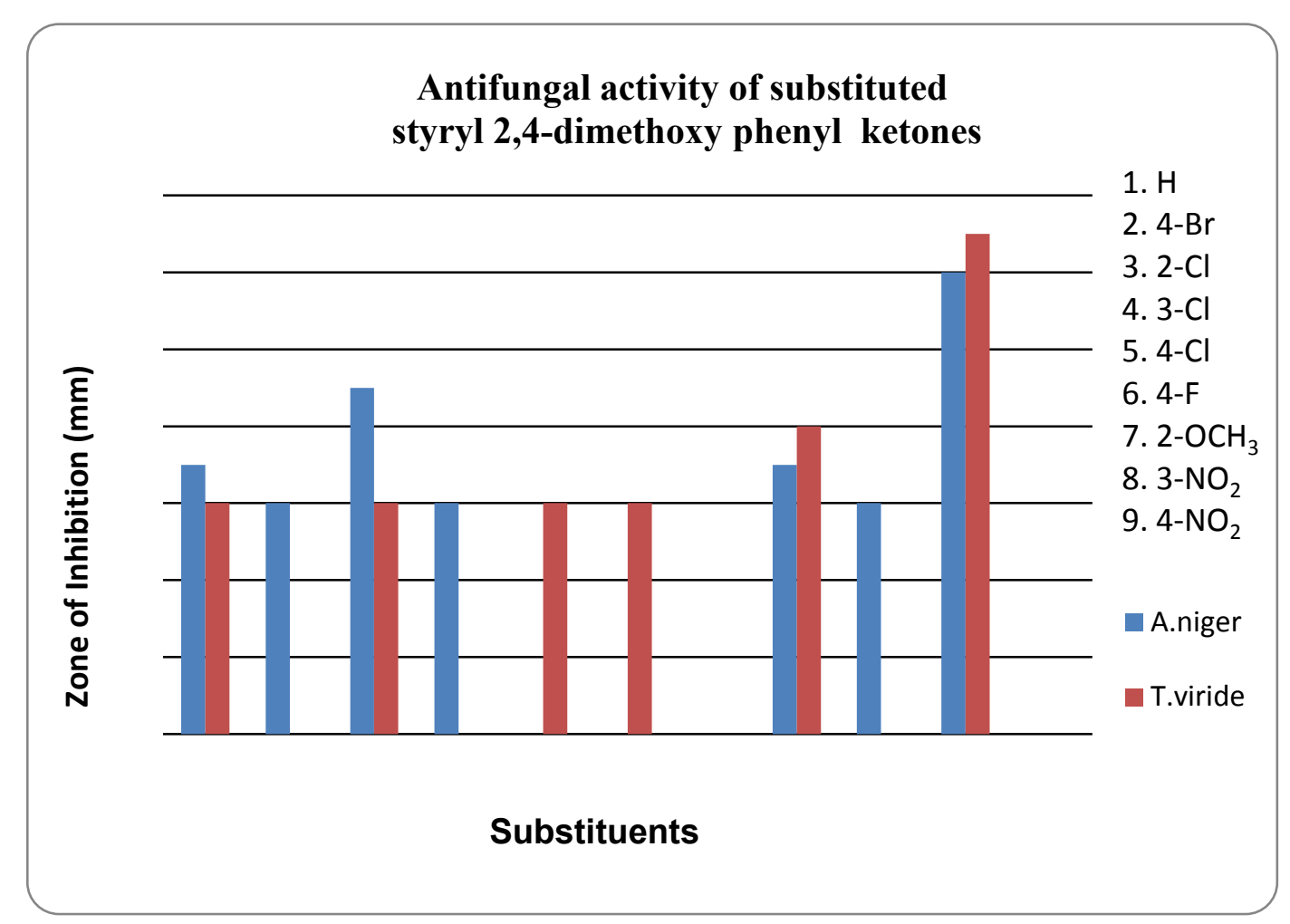

Fig. 6. Antifungal activity of substituted styryl 2,4-dimethoxy phenyl ketones-clustered column chart.

\section{CONCLUSIONS}

A series of 2,4-dimethoxy phenyl chalcones have been synthesized by Crossed-Aldol condensation. The spectral data of these chalcones have been correlated with Hammett sigma constants, $\mathrm{F}$ and $\mathrm{R}$ parameters using single and multi-linear regression analysis. From the results of statistical analysis, the effects of substituents on the spectral group frequencies have been discussed. The anti-microbial activities of these chalcones have been studied.

\section{Acknowledgement}

The authors wished to thank DST NMR Facility, SAIF, IIT, Madras, for recording NMR spectra of all compounds.

\section{References}

[1] Thirunarayanan G., Vanangamudi G., Subramanian M., Organic Chem: An Indian J. 9(1) (2013) 1-16.

[2] Janaki P., Sekar K. G., Thirunarayanan G., J. Saudi Chem. Soc. 2013, Accepted and in press. DOI: org/10.1016/j.jscs.2012.11.013.

[3] Holla B. S., Akberali P. M., Shivananda M. K., IL Farmaco 55 (2000) 256-261.

[4] Venkat Reddy G., Maitraie D., Narsaiah B., Rambabu Y., ShanthanRao P., Synth. Commun 31(18) (2004) 2882-2884.

[5] Orsini F., Sello G., Fumagalli T., Synlett. (2006), 1717-1718. 
[6] Vanangamudi G., Subramanian S., Jayanthi P., Arulkumaran R., Kamalakkannan D., Thirunarayanan G., Arab. J. Chem., 201, DOI: 10.1016/j.arabjc.2010.07.019.

[7] Tran T. D., Park H., Ecker G. F., Thai K. M., 2,3'-Hydroxychalcone Analogues: Synthesis and Structure-PGE2 Inhibitory Activity Relationship, 12 th International Electronic Conference on Synthetic Organic Chemistry (ECSOC-12) 1-30 November 2008 ;

http://www.usc.es/congresos/ecsoc/12/ECSOC12.htm \&http://www.mdpi.org/ecsoc-12 No. C0011, pp 1-7.

[8] Mohan S. B., Behera T. P., Ravi Kumar B. V. V., Int. J. ChemTech. Res. 2(3) 2010 1634-1637.

[9] Thirunarayanan G., Vanangamudi G., Arkivoc. 12 (2006) 58-64.

[10] Thirunarayanan G., Ananthakrishna Nadar P., J. Indian Chem. Soc. 83(11) (2006) $1107-$ 1112 .

[11] Ballini R., Bosica G., Maggi R., Ricciutelli M., Righi P., Sartori G., Sartorio R., Green Chem. 3 (2001) 178-180.

[12] Solhy A., Tahir R., Sebti S. Skouta R., Bousmina M., Zahouily M., Larzek M., Appl. Catal. A: General. 374 (2010) 189-193.

[13] Thirunarayanan G., J. Indian Chem. Soc. 84 (2008) 447-451.

[14] Basaif S. A., Sobahi T. R., Khalil A. K., Hassan M. A., Bulletin of the Korean Chem. Soc. 26(11) (2005) 1677-1681.

[15] Xu Q., Yang Z., Yin D., Zhang F., Catal. Commun. 9(1) (2008) 1579-1582.

[16] Kumar P., Kumar S., Husain K., Kumar A., Bioorg. Med. Chem. 18(14) (2010) $4965-$ 4974.

[17] Zhang Z., Dong Y., W., Wang G. W., Chem. Lett. 32(10) (2003) 966-967.

[18] Thirunarayanan G., IUP. J. Chem. 3(4) (2010) 35-54.

[19] Thirunarayanan G., et al., Elixir Org. Chem. 45 (2012) 7898-7905.

[20] Thirunarayanan G., Mayavel P., Thirumurthy K., Spectrochimica Acta. 91A (2012) 1822.

[21] Sundararajan R., et al., Int. J. Pharm. Chem. Sci. 1(4) (2012) 1657-1677.

[22] Thirunarayanan G., Surya S., Srinivasan S., Vanangamudi G., Sathiyendiran V., Spectrochim. Acta. 75A (2010) 152-156.

[23] Janaki P., Sekar K. G., Thirunarayanan G., Org. Chem: An Indian J. 9(2) (2013) 68-80.

[24] Arulkumaran R., Vijayakumar S., Sundararajan R., Sakthinathan S. P., Kamalakkannan D., Suresh R., Ranganathan K., Vanangamudi G., Thirunarayanan G., International Letters of Chemistry, Physics and Astronomy 4 (2012) 17-38.

[25] Kamalakkannan D, Vanangamudi G, Arulkumaran R, Thirumurthy K, Mayavel P, Thirunarayanan G, Elixir Org. Chem., 46, 2012, 8157-8166.

[26] Sivakumar M., PhrabuSreeneivasan S., Kumar V., Doble M., Bioorg. Med Chem. Lett. 17(10) (2007) 3169-3172. 
[27] Liu X., Go M. L., Bioorg. Med.Chem. 14(1) (2006) 153-163.

[28] Arulkumaran R, et al., IUP J. Chem. 3(1) (2010) 82-98.

[29] Deng J., Sanchez T., Lalith Q. A. M., Bioorg. Med. Chem. 15(14) (2007) 4985-5002.

[30] Bauer A. W., Kirby W. M. M., Sherris J. C., Truck M., Am. J. Clin. Pathol. 45 (1966) 493-496.

[31] Thirunarayanan G., "Fly-ash: $\mathrm{H}_{2} \mathrm{O}$ Assisted Aldol Condensation: Effect of Solvents on the Synthesis of Some Aryl (E) 2-propen-1-ones" Accepted article in Annales, UMCS Chem. J., 2013.

[32] Marquardt F. H., Helv. Chim. Acta. 48 (7) (1965) 1476-1485.

[33] Liu Xiaoling, Bioorg. Med. Chem. 14(1) (2006) 153-163.

[34] Buu-Hoi Ng. Ph., J. Org. Chem. 22 (1957) 193-7.

[35] Vandrewala H. P., J. Univ. Bom Sci: Phys Sci, Math, Biol. Sci. Med. 16 (1948) 43-5.

[36] Kauffmann Hugo, Ber. Dtsch. Chem. Ges. 54B (1921) 795-802.

[37] Swain C. G., Lupton Jr. E. C., J. Am. Chem. Soc. 90(16) (1968) 4328-4337.

[38] Deiva C. M., Pappano N. B., Debattisata N. B., Rev. Microbiol. 29(4) (1998) 307-310.

[39] Thirunarayanan G., J. Saudi Chem. Soc., 2011. DOI:10.1016/j.jscs.2011.12.003.

[40] K. Ranganathan, R. Suresh, D. Kamalakkannan, R. Arulkumaran, R. Sundararajan, S. P. Sakthinathan, S. Vijayakumar, G. Vanangamudi, K. Thirumurthy, P. Mayavel, G. Thirunarayanan, International Letters of Chemistry, Physics and Astronomy 4 (2012) 66-75.

[41] R. Arulkumaran, S. Vijayakumar, R. Sundararajan, S. P. Sakthinathan, D. Kamalakkannan, R. Suresh, K. Ranganathan, P. R. Rajakumar, G. Vanangamudi, G. Thirunarayanan, International Letters of Chemistry, Physics and Astronomy 5 (2013) 21-38.

[42] S. Vijayakumar, R. Arulkumaran, R. Sundararajan, S. P. Sakthinathan, R. Suresh, D. Kamalakkannan, K. Ranganathan, K. Sathiyamoorthy, V. Mala, G. Vanangamudi, G. Thirunarayanan, International Letters of Chemistry, Physics and Astronomy 9(1) (2013) 68-86.

[43] Thirunarayanan G., Sekar K. G., International Letters of Chemistry, Physics and Astronomy 10 (2013) 18-34.

[44] R. Sundararajan, R. Arulkumaran, S. Vijayakumar, D. Kamalakkannan, R. Suresh, S. John Joseph, K. Ranganathan, S. P. Sakthinathan, G. Vanangamudi, G. Thirunarayanan, International Letters of Chemistry, Physics and Astronomy 1 (2014) 67-73. 